Division of Geological \& Geophysical Surveys

RAW-DATA FILE 2007-2

\title{
DATA TABLES RELATED TO GEOLOGY AND GOLD MINERALIZATION IN THE RICHARDSON DISTRICT, EAST-CENTRAL ALASKA
}

by

Garth Erik Graham and Diana Jozwik

$\$ 3.00$

September 2007

THIS REPORT HAS NOT BEEN REVIEWED FOR TECHNICAL CONTENT OR FOR CONFORMITY TO THE EDITORIAL STANDARDS OF DGGS

Released by

STATE OF ALASKA DEPARTMENT OF NATURAL RESOURCES

Division of Geological \& Geophysical Surveys 3354 College Road

Fairbanks, Alaska 99709-3707 



\section{CONTENTS}

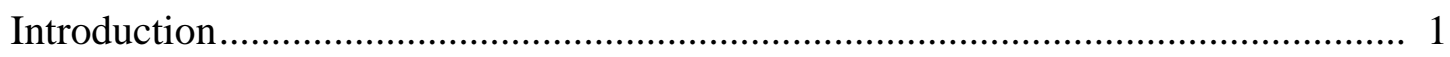

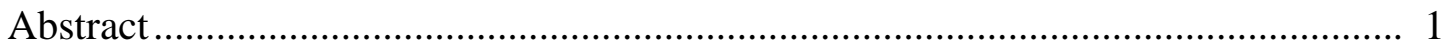

Analytical Methods ............................................................................................ 1

Major Conclusions Taken From Thesis..................................................................... 3

Acknowledgments................................................................................................ 3

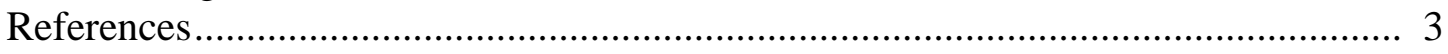

\section{TABLES}

Table 1. List of grab samples, approximate locations, assigned rock type and analyses .................................................................................... 5

2. Modal mineralogy of gneisses ..................................................................13

3. Estimated modal mineral abundances (volume \%) in felsic dike thin sections ........................................................................................13

4. XRF major oxide and normative values for gneiss samples in the Richardson study area ............................................................................14

5. XRF major and minor element results for amphibolite samples

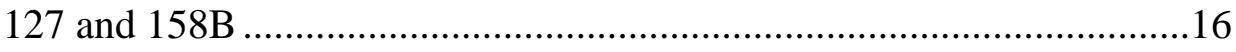

6. XRF major oxide and normative values in percent (\%) for igneous rock samples in the Richardson study area ..............................................17

7. Trace element compositions (in ppm) of igneous rocks from the Richardson study area .........................................................................19

8. Samples employed for geothermometry and geobarometry from Richardson field area ..........................................................................19

9. Averaged microprobe data of mineral assemblages for geothermobarometric calculations ..........................................................20

10. Fluid inclusion samples, locations, and assay values of sampled interval ...........................................................................................23

11. Fluid inclusion data and estimated fluid parameters.................................24

12. Comparison between Bald Knob, Democrat, Ryan Lode fluid

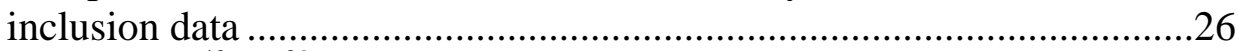

13. Interpreted ${ }^{40} \mathrm{Ar} /{ }^{39} \mathrm{Ar}$ ages (in Ma) for Richardson area samples .............27

14. Ages of geologic events in the Richardson area as suggested by radiometric dating .............................................................................2.

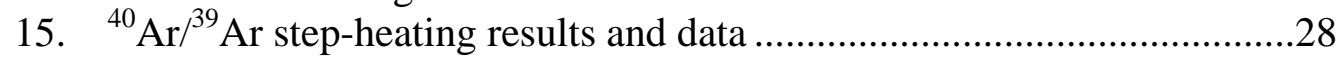

\section{FIGURES}

Figure 1. Location of analyzed samples from the Richardson district, Big Delta B-5 Quadrangle, Alaska .....

2. Location of Bald Knob drill hole samples from the Richardson district, Big Delta B-5 Quadrangle, Alaska

Note: This report (including all analytical data and tables) is available in digital format from the DGGS web site (http://wwwdggs.dnr.state.ak.us) at no charge. The digital data is available as PDF files and Excel spreadsheets. 



\title{
DATA TABLES RELATED TO GEOLOGY AND GOLD MINERALIZATION IN THE RICHARDSON DISTRICT, EAST-CENTRAL ALASKA
}

\author{
by \\ Garth Erik Graham ${ }^{1}$ and Diana Jozwik ${ }^{2}$
}

\section{INTRODUCTION}

The data included in this report were published by Garth Graham as part of his Master of Science thesis at the University of Alaska Fairbanks in 2002 (Graham, 2002). Diana Jozwik compiled the data and selected text for use in this publication to enable easier public access to the data. Data tables shown here were renumbered and formatted to fit this report. Selected informative text was taken from the thesis, merged, and edited for content. More complete discussion of the subjects covered in this report is included in Graham's thesis, available at the University of Alaska Fairbanks (QE84.A4 G73 2002 AK). The abstract from the thesis is given below:

\begin{abstract}
"The Richardson district contains multiple granitic units intruding gneiss and schist. The Bald Knob prospect contains gold-bearing quartz veins with the assemblage Bi-Au-hedleyite and high methane fluid inclusions. These features indicate very low $f_{\mathrm{S} 2}-f_{\mathrm{O} 2}$ conditions, lower than any reported for $90 \mathrm{Ma}$ interior Alaska gold systems, including the nearby Democrat and Buckeye prospects.

The Bald Knob and Democrat Lode prospects returned ${ }^{40} \mathrm{Ar} /{ }^{39} \mathrm{Ar}$ ages of $\sim 104$ and $\sim 90 \mathrm{Ma}$, respectively. Peraluminous dikes possess collisional tectonic signatures and interpreted age of $114 \mathrm{Ma}$. Younger dikes and a $3 \mathrm{~km}^{2}$ granite body possess arc-type compositions and ${ }^{40} \mathrm{Ar} /{ }^{39} \mathrm{Ar}$ ages of $\sim 90 \mathrm{Ma}$.

Garnet-biotite geothermometry on metamorphic rocks indicates low-P regional metamorphism $\left(550-600^{\circ} \mathrm{C} ; 3-4 \mathrm{~kb}\right)$ and vertical movement between adjacent fault blocks. Highest temperatures are in the fault block hosting the Bald Knob prospect, suggesting it represents the deepest mineralization exposure in the area and is most proximal to a causative pluton."
\end{abstract}

Graham's thesis describes the results of geologic mapping and sample analyses from the Richardson district, east-central Alaska, west-central Big Delta B-5 Quadrangle. The objective of the thesis was to create a better geologic map of a portion of the Richardson district and to propose a model for the geological evolution of the Richardson area. During the summers of 1999 and 2000, the study area was mapped on foot; more than 400 field stations were recorded and more than 300 rock samples collected. Location data (in UTM coordinates with a Clark 1866, NAD27, UTM zone 6 projection), rock type, and method of analysis for each sample are shown in table 1. Magnetic susceptibility measurements, included in table 1, were made with a Kappameter model KT-6 magnetic susceptibility meter. Multiple measurements were routinely made on a single sample and the results averaged.

\section{ANALYTICAL METHODS AND RESULTS}

Placer Dome Exploration (PDX) conducted a large-scale soil sampling program in the area in 1999 and 2000. Hand samples were also collected from various locations during concurrent geologic mapping. The geochemical results from these activities were used to determine subsequent core drilling in both 1999 and 2000. Thirty thin sections, 15 doubly-polished from vein samples, 12 polished sections, and one white mica separate were selected from the core drilled by PDX during 1999 and 2000. This core was halved with a rock saw with one-half of each interval sent for assay and the other retained for additional studies.

${ }^{1}$ U.S. Geological Survey, Box 25046 Denver Federal Center MS 973, Denver, Colorado, 80225-0046. Email: ggraham@usgs.gov

${ }^{2}$ Alaska Division of Geological \& Geophysical Surveys, 3354 College Rd., Fairbanks, Alaska 99709-3707 
Modal estimates were conducted on 36 metamorphic and four igneous hand specimens that were etched and stained for quartz, plagioclase, and K-feldspar using the technique of Ruperto and others (1964). K-feldspar assumed a bright yellow color, plagioclase stained red, and quartz remained unstained. Modal estimates were made either by standard point-counting using a minimum of 100 points, or by estimation by comparison to standard abundance charts. Modal abundances are listed in tables 2 and 3.

Major- and minor-oxide analyses were performed by Bondar-Clegg, Incorporated, Vancouver, British Columbia, Canada, on 39 rock samples, including 13 from drill core. Samples were crushed and pulverized in Fairbanks before pulps were shipped to Vancouver. Major- and minor-oxide values were obtained using lithium results, including normative values, are listed in tables 4,5 and 6 . Traceelement analyses for 34 of the samples were performed on pressed pellets at the University of Alaska Fairbanks on splits of the pulverized samples using a Rigaku energy-dispersive XRF and a routine created by Rainer Newberry (described in Cameron, 2000). The results of 16 of the samples are listed in tables 5 and 7. Replicate and secondary standard analyses for commercial and UAF XRF analyses indicate major oxides are accurate to within 2 percent of the amount present, while trace elements are accurate to within 5-10 percent of the amount present (Cameron, 2000).

Microprobe data collected on biotite, garnet, white mica, and K-feldspar were processed using the MacIntosh Geothermobarometry program GTB (Spear and Kohn, 1999). Temperatures were calculated for 12 different garnet-biotite distribution models. Of these 12, those of Perchuk and Lavrenteva (1984) and Kleeman and Reinhardt (1994) with Berman (1990) produced similar, consistent temperatures intermediate to those of other models and were selected as most appropriate for biotite-garnet thermometry. Pressures were estimated based on the calculated temperatures, the aluminosilicate stability diagram of Holdaway (1971), and several compositional-based geobarometers (Spear, 1993). Geothermometry data are presented in table 8 .

Microprobe analyses were performed on six polished sections of gneiss and one polished section from a gold-bearing vein, using the Cameca SX-50 electron microprobe and Probe for Windows software at the University of Alaska Fairbanks. Silicate compositions were measured using a 10 micron beam at 15 nA on wavelength-dispersive spectrometers. Well-characterized natural and synthetic specimens were employed as standards. On-peak counts were collected for 10 seconds and background counts were collected for 5 seconds. Five points were selected for each silicate. These data were ZAF (atomic mass, absorbance, and fluorescence) corrected and poor-quality results were removed from the data set. Opaque minerals, including gold and bismuth, were identified in the gold-bearing vein using the EDS with a standardless analysis routine. Microprobe analyses are presented in table 9.

Fluid inclusion experiments were performed at the USGS facility in Denver, Colorado, using a Linkam heating/freezing stage cooled with liquid nitrogen. All heating and cooling measurements were computer controlled, with standard-based calibrations performed prior to each session. Chips of a section up to $3 \mathrm{~mm}^{2}$ were taken from eight doubly-polished sample sections, 100 to 150 microns thick, and analyzed individually. The chips, attached by glue to the slide, were removed from the section using acetone. Heating and cooling measurements were performed using Linksys, a Windows-driven program, which has a precision of $0.1^{\circ}$ Celsius. The low-temperature measurements included $\mathrm{CO}_{2}$ and clathrate melting temperatures. All measurements except for final homogenization were performed systematically on each inclusion before moving on to the next. After several inclusions were measured, final homogenization temperatures were measured for as many inclusions as possible. Final homogenization observations were made for surrounding inclusions where possible. Once stretching of an inclusion was indicated (by non-repeatable heating experiments), no more homogenization measurements were collected from that chip. Fluid inclusion data are presented in tables 10, 11, and 12.

Five samples were dated using the ${ }^{40} \mathrm{Ar} /{ }^{39} \mathrm{Ar}$ technique, as described in detail by Douglas (1996). The samples were irradiated for 20 megawatt hours in a reactor at McMaster University along with standard sample MMHB-1 with age 513.9 Ma. The standard is used to estimate J, the irradiation parameter and the flux gradient of the reactor. The irradiated samples were then analyzed in the mass spectrometer at the University of Alaska Fairbanks geochronology laboratory, using an ${ }^{40} \mathrm{Ar} /{ }^{39} \mathrm{Ar}$ step heating routine 39-40 days after irradiation. The measured argon isotopes were corrected for mass discrimination as well as for interference of $\mathrm{Ca}, \mathrm{K}$, and $\mathrm{Cl}$ produced from the reactor. Blanks (inlets) were 
run to determine background levels of argon, and measurements were corrected for the background argon. Ages are quoted with a \pm 1 sigma level and calculated using the constants of Steiger and Jaeger (1977). Argon dating results are presented in table 13. The proposed geological events for the Richardson area, based on the radiometric dating, are presented in table 14 . Table 15 lists the ${ }^{40} \mathrm{Ar} /{ }^{39} \mathrm{Ar}$ step-heating results and data.

\section{MAJOR CONCLUSIONS TAKEN FROM THE THESIS}

1. The Richardson area contains two previously unmapped igneous units: the Gold Run Intrusion and felsic dike swarms. The age and composition of the Gold Run Intrusion is indistinguishable from 'classic' 90 Ma Alaskan volcanic arc-related plutons, the closest of which is the Birch Lake pluton. In contrast, the felsic dikes are fine-grained leucocratic granites with significantly older ( 114 Ma) ages with syn-collisional trace-element signatures and a shallow $(\sim 0.5 \mathrm{~km})$ depth of emplacement.

2. Geothermobarometric model temperatures and pressures for gneiss samples fall above the geothermal gradient, ranging from 560 to $620^{\circ} \mathrm{C}$ and 3-4 kbars, respectively, that contrast with similar temperatures but higher pressures recorded in Fairbanks-area rocks. However, these values do not necessarily require different peak metamorphic conditions. Rather, rocks in the Richardson area apparently experienced a younger recrystallization episode at relatively high temperature and low pressure, possibly related to collisional tectonics.

3. Sericite from the selvage of a gold-bearing quartz vein yielded a ${ }^{40} \mathrm{Ar} /{ }^{39} \mathrm{Ar}$ interpreted age of $\sim 105$ Ma, significantly older than the $~ 90$ Ma event age of the Gold Run Intrusion and those commonly associated with most other gold systems in interior Alaska, including the Democrat Dike. Petrography and fluid inclusion studies indicate that the ore-bearing fluid was highly reduced (methane in fluid inclusions) and had low $\mathrm{fS}_{2}$ (lack of sulfide minerals in the presence of native bismuth, hedleyite, and gold). Temperature of formation is estimated between 400 and $500^{\circ} \mathrm{C}$. The mineralogy and fluid inclusion chemistry associated with gold deposition is radically different from the younger Democrat lode deposit. The $\sim 105 \mathrm{Ma}$ age suggests a possibility for a genetic relationship between gold mineralization and emplacement of the felsic dikes.

\section{ACKNOWLEDGMENTS}

We thank Dr. Rainer Newberry of the University of Alaska Fairbanks, whose patience in answering questions and providing advice was immensely valuable.

\section{REFERENCES}

Angus, Selby, Armstrong, Barrie, and Altunin, V.V., 1976, International thermodynamic tables of the fluid state; Volume 3, Carbon dioxide: Oxford, Pergamon Press, 385 p.

Berman, R.G., 1990, Mixing properties of Ca-Mg-Fe-Mn garnets: American Mineralogist, v. 75, p. 328334.

Brown, P.E., and Lamb, W.M., 1989, P-V-T properties of fluids in the system $\mathrm{H}_{2} \mathrm{O} \pm \mathrm{CO}_{2} \pm \mathrm{NaCL}$; New graphical presentations and implications for fluid inclusion studies: Geochimica et Cosmochimica Acta, v. 53, no. 6, p. 1209-1221.

Bundtzen, T.K., and Reger, R.D., 1977, The Richard lineament - a structural control for gold deposits in the Richardson mining district, interior Alaska in Alaska Geological \& Geophysical Surveys, Short Notes on Alaskan Geology - 1977: Alaska Geological \& Geophysical Surveys Geologic Report 55, p. 29-34.

Burruss, R.C., 1981, Analysis of phase equilibria in C-O-H-S fluid inclusions, in Hollister, L.S., and Crawford, M.L., eds., Fluid inclusions; applications to petrology: Mineralogical Association of Canada Short Course, Royal Ontario Museum, v. 6, p. 39-74.

Cameron, C.E., 2000, Fault-hosted Au mineralization, Ester Dome, Alaska: Fairbanks, Alaska, University of Alaska Fairbanks, M.S. Thesis, 115 p. 
Douglas, T.A., 1996, Metamorphic histories of the Chatanika eclogite and Fairbanks schist within the Yukon-Tanana terrane, Alaska, as revealed by electron microprobe thermobarometry and ${ }^{40} \mathrm{Ar} /{ }^{39} \mathrm{Ar}$ single grain dating: Fairbanks, Alaska, University of Alaska Fairbanks, M.S. thesis, 240 p.

Foster, H.L., Albert, N.R.D., Griscom, Andrew, Hessin, T.D., Menzie, W.D., Turner, D.L., and Wilson, F.H., 1979, The Alaskan Mineral Resource Assessment Program; Background information to accompany folio of geologic and mineral resource maps of the Big Delta Quadrangle, Alaska: U.S. Geological Survey Circular 783, 19 p.

Ganguly, Jibamitra, and Saxena, S.K., 1984, Mixing properties of aluminosilicate garnets; Constraints from natural and experimental data, and applications to geothermo-barometry: American Mineralogist, v. 69, no. 1-2, p.88-97.

Ghent, E.D., and Stout, M.Z., 1981, Geobarometry and geothermometry of plagioclase-biotite-garnetmuscovite assemblages: Contributions to Mineralogy and Petrology, v. 76, no. 1, p. 92-97.

Graham, G.E., 2002, Geology and gold mineralization of the Richardson district, east-central Alaska: Fairbanks, Alaska, University of Alaska Fairbanks, M.S. Thesis, 150 p.

Hodges, K.V., and Crowley, P.D., 1985, Error estimation and empirical geothermobarometry for pelitic systems: American Mineralogist, v. 70, no. 7-8, p. 702-709.

Hodges, K.V., and Spear, F.S., 1982, Geothermometry, geobarometry and the $\mathrm{Al}_{2} \mathrm{SiO}_{5}$ triple point at Mt. Moosilauke, New Hampshire: American Mineralogist, v. 67, no. 11-12, p. 1118-1134.

Hoisch, T.D., 1990, Empirical calibration of six geobarometers for the mineral assemblage quartz + muscovite + biotite + plagioclase + garnet: Contributions to Mineralogy and Petrology, v. 104, no. 2, p. 225-234.

Holdaway, M.J., 1971, Stability of andalusite and the aluminum silicate phase diagram: American Journal of Science, v. 271, p. 97-131.

Kennedy, G.C., 1954, Pressure-volume-temperature relations in $\mathrm{CO}_{2}$ at elevated temperatures and pressures: American Journal of Science, v. 252, no. 4, p. 225-241.

Kleemann, Ulrich, and Reinhardt, Juergen, 1994, Garnet-biotite thermometry revisited; The effect of $\mathrm{Al}^{\mathrm{VI}}$ and $\mathrm{Ti}$ in biotite: European Journal of Mineralogy, v. 6, p. 925-941.

McCoy, D.T., Newberry, R.J., Layer, P.W., DiMarchi, J.J., Bakke, A.A., Masterman, J.S., and Minehane, D.L., 1997, Plutonic-related gold deposits of interior Alaska: Economic Geology Monographs, v. 9, p.191-241.

McDougall, Ian, and Harrison, T.M., 1988, Geochronology and thermochronology by the (super 40) Ar/ (super 39) Ar method: Oxford Monographs on Geology and Geophysics, v. 9, 212 p.

Pakhomova, V., Belyaeva, V., and Tishkin, B., 1995, Results of a thermobarometric study of hydrothermal fluids and magmatic system at the Democrat lode deposit, Richardson district, Alaska, in Bundtzen, T.K., Fonseca, A.L., and Mann, Roberta, eds., The Geology and Mineral Deposits of the Russian Far East: Alaska Miners Association Special Symposium, v. 1, Glacier House Publications, Anchorage, Alaska, p. 128-133.

Newton, R.C., and Haselton, H.T., 1981, Thermodynamics of the garnet-plagioclase- $\mathrm{Al}_{2} \mathrm{SiO}_{5}-$ quartz geobarometer, in Newton, R.C., Navrotsky, Alexandra, and Wood, B.J., eds., Thermodynamics of minerals and melts: New York, Springer-Verlag, p. 131-147.

Perchuk, L.L., and Lavrenteva, I.V., 1983, Experimental investigation of exchange equilibria in the system cordierite-garnet-biotite, in Saxena, S.K., ed., Kinetics and equilibrium in mineral reactions: New York, Springer-Verlag, p. 199-240.

Roedder, E.R., 1984, Fluid inclusions: Reviews in Mineralogy, v. 12, 644 p.

Ruperto, V.L., Stevens, R.E., and Norman, M.B., 1964, Staining of plagioclase feldspars and other minerals with FD\&C Red No. 2: U.S. Geological Survey Professional Paper 501-B, p. B152-B153.

Spear, F.S., 1993, Metamorphic phase equilibria and pressure-temperature-time paths: Washington, D.C., Mineralogical Society of America, 799 p.

Spear, F.S., and Kohn, M.J., 1999, Thermobarometry (GTB), Version 2.1

Steiger, R.H., and Jaeger, Emilie, comps., 1977, Subcommission on geochronology; Convention of the use of decay constants in geo- and cosmochronology: Earth and Planetary Science Letters, v. 36, no. 3, p. 359-362. 
Table 1. List of grab samples, approximate locations, assigned rock types and analyses. T.S. $=$ thin section, P.S. $=$ polished section, WRA = whole rock analysis, Geotherm = microprobe, geothermobarometric analysis, FI = fluid inclusion study, $\mathrm{Ar} / \mathrm{Ar}={ }^{40} \mathrm{Ar} /{ }^{39} \mathrm{Ar}$ radiometric dating. Blank sample\# indicates observations were recorded but no sample was collected. Keys to rock unit abbreviations are listed at end of this table on page 12

\begin{tabular}{|c|c|c|c|c|c|c|c|c|c|c|}
\hline Sample\# & UTMX & UTMY & Rock type & $\begin{array}{l}\text { Mag. Susc. } \\
\left(* 10^{\wedge}-3 \text { SI }\right)\end{array}$ & T.S. & P.S. & WRA & Geotherm & FI & $\mathrm{Ar} / \mathrm{Ar}$ \\
\hline 1 & 537115 & 7137230 & MTR & 0.02 & & & & & & \\
\hline 2 & 537015 & 7137040 & MTR & & & & & & & \\
\hline 3 & 537015 & 7137000 & BLG & & & & & & & \\
\hline 4 & 536900 & 7136900 & MTR & & & & & & & \\
\hline 5 & 536900 & 7136900 & MI & & & & & & & \\
\hline 6 & 536875 & 7136850 & MTR & & & & & & & \\
\hline 7 & 536830 & 7136780 & MI & & & & & & & \\
\hline 8 & 536940 & 7136715 & MTR & & & & & & & \\
\hline 9 & 537200 & 7136920 & BLG & & & & & & & \\
\hline 10 & 537220 & 7137020 & BLG & & & & & & & \\
\hline 11 & 537400 & 7137000 & MI & 0.25 & & & & & & \\
\hline 12 & 537400 & 7137050 & MI & & & & & & & \\
\hline 14 & 537100 & 7137300 & MTR & & & & & & & \\
\hline 15 & 537100 & 7137600 & MTR & & & & & & & \\
\hline 16 & 537200 & 7135950 & MTR & 0.15 & & & & & & \\
\hline 17 & 537050 & \begin{tabular}{|l|}
7135850 \\
\end{tabular} & MI & 0.14 & & & & & & \\
\hline 17 & 537050 & 7135850 & MTR & & & & & & & \\
\hline 18 & 537030 & 7135830 & QFR & & & & & & & \\
\hline 19 & 537100 & 7135800 & HFL & 0.2 & & & & & & \\
\hline 19 & 537100 & 7135800 & BLG & 0.02 & & & & & & \\
\hline 20 & 537150 & 7135800 & MI & & & & & & & \\
\hline 21 & 537200 & 7135700 & MTR & & & & & & & \\
\hline 22 & 537170 & 7135500 & GN & & & & & & & \\
\hline 23 & 536650 & 7135830 & MTR & 0.1 & & & & & & \\
\hline 24 & 536450 & 7135650 & MTR & & & & & & & \\
\hline 25 & 536600 & 7135600 & BLG & & & & & & & \\
\hline 26 & 536630 & 7134590 & MTR & 0.11 & & & & & & \\
\hline 27 & 536850 & \begin{tabular}{|l|}
7135350 \\
\end{tabular} & MTR & 0.15 & & & & & & \\
\hline 28 & 536950 & 7135350 & GN & & & & & & & \\
\hline 29 & 537050 & 7135200 & BLG & & & & & & & \\
\hline 30 & 537100 & 7135100 & MTR & 0.12 & & & & & & \\
\hline 31 & 537200 & 7135150 & BLG & 0.15 & & & & & & \\
\hline 32 & 537750 & 7135300 & MTR & 0.14 & & & & & & \\
\hline 33 & 536590 & 7136150 & MTR & & & & & & & \\
\hline 34 & 536450 & 7135900 & FGN & & & & & & & \\
\hline 35 & 536300 & 7135450 & MI & 0.12 & & & & & & \\
\hline 36 & 535650 & 7134850 & SKN & 0.18 & & & & & & \\
\hline 37 & 535450 & 7134300 & QFR & 0 & & & & & & \\
\hline 38 & 535750 & 7134400 & GN & & & & & & & \\
\hline 39 & 535850 & 7134450 & $\mathrm{SCH}$ & & & & & & & \\
\hline 40 & 536050 & 7134350 & SGN & & & & & & & \\
\hline 41 & 536050 & 7134400 & GN & & & & & & & \\
\hline 42 & 536050 & 7134450 & FBQ & & & & & & & \\
\hline 43 & 536200 & 7134600 & QFR & & & & & & & \\
\hline 44 & 536200 & 7134800 & GN,HFL & & & & & & & \\
\hline 45 & 536250 & 7134850 & HFL, GN & $0.3,0.15$ & & & & & & \\
\hline
\end{tabular}


Table 1 continued. List of grab samples, approximate locations, assigned rock types and analyses.

\begin{tabular}{|c|c|c|c|c|c|c|c|c|c|c|}
\hline Sample\# & UTMX & UTMY & Rock type & $\begin{array}{l}\text { Mag. Susc. } \\
\left(* 10^{\wedge}-3 \text { SI }\right)\end{array}$ & T.S. & P.S. & WRA & Geotherm & FI & $\mathrm{Ar} / \mathrm{Ar}$ \\
\hline 46 & 536250 & 7134850 & FGN & & & & & & & \\
\hline 47 & 536250 & 7134850 & HFL & & & & & & & \\
\hline 48 & 536200 & 7134950 & QFR & & & & & & & \\
\hline 49 & 536200 & 7134950 & SGN & & & & & & & \\
\hline 50 & 536250 & 7135500 & BLG & & & & & & & \\
\hline 51 & 536300 & 7135350 & MTR & & & & & & & \\
\hline 52 & 536300 & 7135150 & GN & & & & & & & \\
\hline 53 & 536300 & 7135000 & INT & & & & & & & \\
\hline 54 & 536300 & 7134900 & GN & 0.03 & & & & & & \\
\hline 55 & 536330 & 7134940 & $\mathrm{GN}$ & & & & & & & \\
\hline 56 & 536300 & 7134850 & GN & & & & & & & \\
\hline 57 & 536300 & 7134780 & PEG & & & & & & & \\
\hline 58 & 536250 & 7134750 & QFR & 0.01 & & & & & & \\
\hline 59 & 536200 & 7134700 & QTZ & & & & & & & \\
\hline 60 & 536200 & 7134700 & HFL & & & & & & & \\
\hline 61 & 536300 & 7134700 & FGN & & & & & & & \\
\hline 62 & 536300 & 7134600 & GN & & & & & & & \\
\hline 63 & 536500 & 7134700 & GN & & & & & & & \\
\hline 64 & 536450 & 7134750 & BLG & & & & & & & \\
\hline 65 & 536050 & 7135100 & AMPH, GN &,--- 0.15 & & & & & & \\
\hline 66 & 535950 & 7135000 & SGN & & & & & & & \\
\hline 67 & 535830 & 7134975 & SGN & & & & & & & \\
\hline 68 & 535600 & 7134850 & SKN & & & & & & & \\
\hline 69 & 535450 & 7134750 & SGN & 0.2 & & & & & & \\
\hline 70 & 535450 & 7134700 & SGN & & & & & & & \\
\hline 71 & 535450 & 7134450 & GGE & & & & & & & \\
\hline 72 & 535450 & 7134450 & QTZ & & & & & & & \\
\hline 73 & 535450 & 7134450 & QFT & & $\mathrm{x}$ & & $\mathrm{x}$ & & & \\
\hline 74 & 535450 & 7134450 & ALT & & & & & & & \\
\hline 75 & 535500 & 7134300 & ALT & & & & & & & \\
\hline 76 & 535650 & 7134250 & SGN & & & & & & & \\
\hline 78 & 535350 & 7134260 & $\mathrm{SCH}$ & & & & & & & \\
\hline 79 & 535000 & 7134130 & PEG & & & & & & & \\
\hline 80 & 534900 & 7134250 & GN & & & & & & & \\
\hline 81 & 534800 & 7134250 & GN & 0.2 & $\mathrm{x}$ & & $\bar{x}$ & & & \\
\hline 82 & 534650 & 7134250 & SGN & 0.06 & & & & & & \\
\hline 83 & 534350 & 7134150 & SGN & 0.15 & & & & & & \\
\hline 84 & 533800 & 7134250 & GN & 0 & & & & & & \\
\hline 85 & 533800 & 7134450 & MI & 0.1 & & & & & & \\
\hline 86 & 533800 & 7134550 & BLG & & & & & & & \\
\hline 87 & 533850 & 7134550 & BLG & & & & & & & \\
\hline 88 & 534000 & 7134650 & BLG & & & & & & & \\
\hline 89 & 534100 & 7134850 & GN & & & & & & & \\
\hline \begin{tabular}{|l|}
90 \\
\end{tabular} & 534100 & 7134850 & BLG & & & & & & & \\
\hline 91 & 534200 & 7135200 & MI & 0.1 & & & & & & \\
\hline 92 & 534200 & 7135400 & MTR & & & & & & & \\
\hline 93 & 534150 & 7135650 & MI & 0.09 & & & & & & \\
\hline 94 & 534650 & 7135950 & $\overline{Q F R}$ & & & & & & & \\
\hline \begin{tabular}{|l}
95 \\
\end{tabular} & 534850 & 7136100 & ALT & & & & & & & \\
\hline
\end{tabular}


Table 1 continued. List of grab samples, approximate locations, assigned rock types and analyses.

\begin{tabular}{|c|c|c|c|c|c|c|c|c|c|c|}
\hline Sample\# & UTMX & UTMY & Rock type & $\begin{array}{l}\text { Mag. Susc. } \\
\left(* 10^{\wedge}-3 \text { SI }\right)\end{array}$ & T.S. & P.S. & WRA & Geotherm & FI & Ar/Ar \\
\hline 96 & 535300 & 7136450 & $\overline{\mathrm{ALT}}$ & & & & & & & \\
\hline 97 & 535400 & 7136400 & MI & 0.12 & & & & & & \\
\hline \multirow[t]{3}{*}{98} & 535750 & 7136650 & MI & & & & & & & \\
\hline & 536000 & 7136700 & BLG & & & & & & & \\
\hline & 536350 & 7136500 & MI & & & & & & & \\
\hline 100 & 535650 & 7137300 & MI & & & & & & & \\
\hline 101 & 535550 & 7137400 & MI & & & & & & & \\
\hline 102 & 535500 & 7137450 & MI & & & & & & & \\
\hline 103 & 535150 & 7137900 & MI & & & & & & & \\
\hline 104 & 535000 & 7138000 & MI & & & & & & & \\
\hline 105 & 534750 & 7138300 & SGN & & & & & & & \\
\hline 106 & 534750 & 7138400 & BLG & & & & & & & \\
\hline \multirow[t]{2}{*}{107} & 534750 & 7138500 & GN & & & & & & & \\
\hline & 534850 & 7138550 & SGN & & & & & & & \\
\hline \begin{tabular}{|l|}
108 \\
\end{tabular} & 534350 & 7138750 & GN & & & & & & & \\
\hline 109 & 534250 & 7138700 & MI & & & & & & & \\
\hline 110 & 534250 & 7138700 & SGN & & & & & & & \\
\hline 111 & 534250 & 7138750 & SGN & & & & & & & \\
\hline 112 & 529900 & 7139200 & GN & & $\mathrm{x}$ & & $\mathrm{X}$ & & & \\
\hline 113 & 529900 & 7139180 & GN & & & & & & & \\
\hline 114 & 528950 & 7139150 & GN & & & & & & & \\
\hline 115 & 528800 & 7139050 & QFR & & & & & & & \\
\hline 116 & 528750 & 7139100 & ALT & & & & & & & \\
\hline 117 & 528750 & 7139050 & FGN & & & & & & & \\
\hline 118 & 528650 & 7138950 & GN & & $\mathrm{X}$ & $\mathrm{x}$ & $\mathrm{x}$ & & & \\
\hline 119 & 528500 & 7138850 & $\overline{A L T}$ & & & & & & & \\
\hline 120 & 528200 & 7138750 & ALT & & & & & & & \\
\hline 121 & 528150 & 7138750 & RHY & & & & & & & \\
\hline 122 & 530250 & 7139050 & GN & & $\mathrm{x}$ & $\mathrm{x}$ & & $\mathrm{x}$ & & \\
\hline 123 & 530500 & 7139050 & GN & & $\mathrm{x}$ & $\mathrm{x}$ & $\mathrm{x}$ & & & \\
\hline \begin{tabular}{|l|}
124 \\
\end{tabular} & 530650 & 7139000 & GN & & $\mathrm{x}$ & & $\mathrm{x}$ & & & \\
\hline 125 & 530750 & 7139000 & GN+QFR & & $\mathrm{x}$ & & $\mathrm{X}$ & & & $\mathrm{x}$ \\
\hline 126 & 530860 & 7138960 & GN & & & & & & & \\
\hline 127 & 530860 & 7138960 & AMPH & & & & $\mathrm{X}$ & & & \\
\hline 128 & 530860 & 7138960 & GN & & $\mathrm{x}$ & & $\mathrm{X}$ & & & \\
\hline 129 & 531150 & 7139100 & GN & & & & $\mathrm{x}$ & & & \\
\hline 130 & 531350 & 7139400 & QFR & & & & & & & \\
\hline 131 & 531350 & 7139450 & GN & & & & & & & \\
\hline \multirow[t]{2}{*}{132} & 531350 & 7139450 & QFR & & & & & & & \\
\hline & 531350 & 7139450 & QFR & & & & & & & \\
\hline \multirow[t]{2}{*}{133} & 531600 & 7140200 & QFR & & & & & & & \\
\hline & 531600 & 7140200 & SGN & & & & & & & \\
\hline \multirow[t]{3}{*}{134} & 531700 & 7140200 & ALT & & & & & & & \\
\hline & 531700 & 7140200 & RHY & & & & & & & \\
\hline & 531700 & 7140200 & FGN & & & & & & & \\
\hline 135 & 531650 & 7140250 & SGN & & & & $\mathrm{x}$ & & & \\
\hline 136 & 531750 & 7140500 & QFR & & & & & & & \\
\hline 137 & 531750 & 7140500 & GN & & $\bar{x}$ & & $\mathrm{x}$ & & & \\
\hline 138 & 531900 & 7140800 & QFR & & & & & & & \\
\hline
\end{tabular}


Table 1 continued. List of grab samples, approximate locations, assigned rock types and analyses.

\begin{tabular}{|c|c|c|c|c|c|c|c|c|c|c|}
\hline Sample\# & UTMX & UTMY & Rock type & $\begin{array}{l}\text { Mag. Susc. } \\
\left({ }^{*} 10^{\wedge}-3 \text { SI }\right)\end{array}$ & T.S. & P.S. & WRA & Geotherm & FI & $\mathbf{A r} / \mathbf{A r}$ \\
\hline \multirow[t]{2}{*}{139} & 532050 & 7141000 & $\overline{Q F R}$ & & & & & & & \\
\hline & 532350 & 7140700 & QFR & & & & & & & \\
\hline 140 & 532900 & 7140450 & QFR & & $\mathrm{x}$ & & $\mathrm{x}$ & & & \\
\hline \multirow[t]{3}{*}{141} & 533000 & 7140400 & GN & & & & & & & \\
\hline & 533000 & 7140400 & QFR & & & & & & & \\
\hline & 533000 & \begin{tabular}{|l|}
7140400 \\
\end{tabular} & PEG & & & & & & & \\
\hline \multirow[t]{2}{*}{142} & 533000 & 7140400 & GN & & $\mathrm{x}$ & & $\mathrm{x}$ & & & \\
\hline & 533050 & 7140300 & QFR & & & & & & & \\
\hline 143 & 533100 & 7140350 & QFR & & $\mathrm{x}$ & & $\mathrm{x}$ & & & $\mathrm{x}$ \\
\hline 144 & 533100 & 7140050 & GN & & & & & & & \\
\hline 145 & 533050 & 7139750 & QFR & & & & & & & \\
\hline 146 & 533050 & \begin{tabular}{|l|l}
7139700 \\
\end{tabular} & QFR & & & & & & & \\
\hline 147 & 533000 & 7139350 & GN & & & & & & & \\
\hline 148 & 532750 & 7139100 & QFR & & & & & & & \\
\hline 149 & 532600 & 7138950 & GN & & & & & & & \\
\hline 150 & 532600 & 7138850 & GN & & & & & & & \\
\hline 151 & 532500 & 7138350 & QFR & & & & & & & \\
\hline 152 & 532350 & 7138300 & GN & & & & & & & \\
\hline 153 & 532300 & 7138200 & GN & & & & & & & \\
\hline 154 & 532000 & 7138200 & QFR & & & & & & & \\
\hline 155 & 531900 & 7138200 & SGN & & & & & & & \\
\hline 156 & 531500 & 7138600 & AMPH & & & & & & & \\
\hline 157 & 531650 & 7137500 & $\mathrm{GN}$ & & & & & & & \\
\hline 158 & 531650 & 7137350 & \begin{tabular}{|c|} 
QFR+GN+ \\
AMPH \\
\end{tabular} & & $\mathrm{X}, \mathrm{X}$ & & $\mathrm{x}$ & & & \\
\hline 159 & 531200 & 7136800 & SGN & & & & & & & \\
\hline 160 & 537400 & 7133650 & GN & & & & & & & \\
\hline 161 & 537350 & 7134050 & SGN & & & & & & & \\
\hline 163 & 537650 & 7135500 & MTR & & & & & & & \\
\hline 164 & 537350 & 7136100 & MTR & & & & & & & \\
\hline 165 & 537550 & 7136050 & BLG & & & & & & & \\
\hline 166 & 537550 & 7135900 & MTR & & & & & & & \\
\hline 167 & 537630 & 7135865 & GN & & & & & & & \\
\hline 168 & 537800 & 7135830 & GN & & & & & & & \\
\hline 169 & 537900 & 7135700 & GN & & & & & & & \\
\hline 170 & 537850 & 7135500 & BLG & & & & & & & \\
\hline 171 & 538000 & 7135400 & MTR & & & & & & & \\
\hline 172 & 538100 & 7135250 & GN & & & & & & & \\
\hline 173 & 538050 & 7135050 & MTR & & & & & & & \\
\hline 174 & 538040 & 7135000 & GN & & & & & & & \\
\hline 175 & 537750 & 7134450 & QFR & & & & & & & \\
\hline 176 & 537525 & 7134300 & MI & & & & & & & \\
\hline 177 & 538300 & 7138000 & MTR & & & & & & & \\
\hline 178 & 531500 & 7134500 & MI & & & & & & & \\
\hline 179 & 531550 & 7134450 & GN & & & & & & & \\
\hline 180 & 531550 & 7135150 & GN & & & & & & & \\
\hline 99GG001 & 525760 & 7141860 & PEG & -0.03 & & & & & & \\
\hline 99GG002 & 526100 & 7142150 & MI & -0.02 & & & & & & \\
\hline 99GG003 & 526900 & \begin{tabular}{|l|l}
7145500 \\
\end{tabular} & $\overline{\text { QFR }}$ & 0 & $\mathrm{x}$ & & & & & \\
\hline
\end{tabular}


Table 1 continued. List of grab samples, approximate locations, assigned rock types and analyses.

\begin{tabular}{|c|c|c|c|c|c|c|c|c|c|c|}
\hline Sample\# & UTMX & UTMY & Rock type & $\begin{array}{l}\text { Mag. Susc. } \\
\left(* 10^{\wedge}-3 \text { SI }\right)\end{array}$ & T.S. & P.S. & WRA & Geotherm & FI & Ar/Ar \\
\hline 99GG004 & 526600 & 7143900 & SGN & 0.08 & & & & & & \\
\hline 99GG005 & 527700 & 7143350 & QFR & 0.03 & & & & & & \\
\hline 99GG006 & 527525 & 7141700 & MI & 0.08 & & & & & & \\
\hline 99GG007 & 527890 & 7141290 & PEG & -0.02 & & & & & & \\
\hline 99GG008 & 528200 & 7140040 & MI & 0.1 & $\mathrm{x}$ & $\mathrm{x}$ & & & & \\
\hline 99GG009 & 527840 & 7140035 & MI & 0.05 & $\mathrm{x}$ & $\mathrm{x}$ & & & & \\
\hline 99GG010 & 528000 & 7140000 & MI & 0.06 & & & & & & \\
\hline 99GG011 & 527500 & 7139950 & MI & 0.06 & & & & & & \\
\hline 99GG012 & 527350 & 7139870 & MI & 0.15 & & & & & & \\
\hline 99GG013 & 527350 & 7139800 & GN & 0.1 & $\mathrm{x}$ & & & & & \\
\hline 99GG014 & 528200 & 7140400 & $\mathrm{GN}$ & 0.02 & & & & & & \\
\hline 99GG015 & 528825 & 7139670 & GN & 0.02 & & & & & & \\
\hline 99GG016 & 528360 & 7141760 & QFR & 0.02 & & & & & & \\
\hline 99GG017 & 528400 & 7141750 & GN & 0.06 & & & & & & \\
\hline 99GG018 & 528410 & 7141750 & MGN & 0.12 & $\mathrm{x}$ & $\mathrm{x}$ & & & & \\
\hline 99GG019 & 528300 & 7140040 & $\mathrm{MI}$ & 0.1 & & & & & & \\
\hline 99GG020 & 528400 & 7141900 & QFR & 0.02 & & & & & & \\
\hline 99GG021 & 528460 & 7140900 & MI & -0.2 & & & & & & \\
\hline 99GG022 & 528200 & 7141060 & QFR & 0 & & & & & & \\
\hline 99GG023 & 528400 & 7141900 & AGN & -0.02 & & & & & & \\
\hline \begin{tabular}{|l|} 
99GG024 \\
\end{tabular} & 528460 & 7142640 & SGN & 0.15 & $\mathrm{x}$ & $\mathrm{x}$ & $\mathrm{x}$ & & & \\
\hline 99GG025 & 528270 & 7140760 & GN & 0.02 & & & & & & \\
\hline 99GG026 & 528350 & 7140000 & GN & 0.05 & $\mathrm{x}$ & & & & & \\
\hline \begin{tabular}{|l|} 
99GG027 \\
\end{tabular} & 528110 & 7141060 & SGN & 0.1 & & & & & & \\
\hline 99GG028 & 528150 & 7141340 & QTZ & 0 & & & & & & \\
\hline 99GG029 & 528400 & 7140270 & QFR & 0 & & & & & & \\
\hline 99GG030 & 528360 & 7142050 & QFR & 0 & & & & & & \\
\hline 99GG031 & 528490 & 7140274 & GN & 0.08 & $\mathrm{x}$ & & $\mathrm{X}$ & & & \\
\hline 99GG032 & 528110 & 7141060 & ALT & 0 & & & & & & \\
\hline 99GG033 & 528750 & 7140800 & MI & 0 & & & & & & \\
\hline \begin{tabular}{|l|} 
99GG034 \\
\end{tabular} & 528480 & 7140780 & SGN & 0.06 & & & & & & \\
\hline 99GG035 & 528290 & 7140750 & RHY & 0 & & & & & & \\
\hline 99GG036 & 530520 & 7140200 & $\overline{Q F R}$ & -0.02 & & & & & & \\
\hline 99GG037 & 528400 & 7141750 & QFR & -0.05 & & & & & & \\
\hline 99GG038 & 528825 & 7139670 & ALT & $\begin{array}{l}-0.03 \\
\end{array}$ & & & & & & \\
\hline \begin{tabular}{|l|} 
99GG039 \\
\end{tabular} & 529500 & 7140700 & MTR & 0 & & & & & & \\
\hline 99GG040 & 529050 & 7139300 & GN & 0 & $\mathrm{X}$ & $\mathrm{x}$ & $\mathrm{X}$ & & & \\
\hline 99GG041 & 529550 & 7140700 & QFR & 0.04 & & & & & & \\
\hline 99GG042 & 529800 & 7137900 & MI & -0.02 & & & & & & \\
\hline 99GG043 & 530500 & 7140000 & GN & 0.08 & $\mathrm{x}$ & & & & & \\
\hline 99GG044 & 529730 & 7139500 & BLG & 0.02 & & & & & & \\
\hline 99GG045 & 529800 & 7138700 & GN & 0.01 & & & & & & \\
\hline \begin{tabular}{|l|} 
99GG046 \\
\end{tabular} & 530850 & 7139630 & AMPH & 0.6 & & & & & & \\
\hline 99GG047 & 530270 & 7140450 & PEG & $\begin{array}{l}-0.03 \\
\end{array}$ & & & & & & \\
\hline 99GG048 & 530300 & 7142000 & QFR & 0 & & & & & & \\
\hline 99GG049 & 530725 & 7138900 & QTZ & 0 & & & & & & \\
\hline 99GG050 & 530530 & 7140230 & GN & 0 & & & & & & \\
\hline 99GG051 & 530530 & 7140230 & $\overline{\text { QFR }}$ & 0 & & & & & & \\
\hline 99GG052 & 530775 & 7138900 & QTZ & 0 & & & & & & \\
\hline
\end{tabular}


Table 1 continue. List of grab samples, approximate locations, assigned rock types and analyses.

\begin{tabular}{|c|c|c|c|c|c|c|c|c|c|c|}
\hline Sample\# & UTMX & UTMY & Rock type & $\begin{array}{l}\text { Mag. Susc. } \\
\left(* 10^{\wedge}-3 \text { SI }\right)\end{array}$ & T.S. & P.S. & WRA & Geotherm & FI & Ar/Ar \\
\hline 99GG053 & 530350 & 7140550 & $\overline{\mathrm{AGN}}$ & 0 & & & & & & \\
\hline 99GG054 & 531300 & 7137100 & RHY & -0.05 & & & & & & \\
\hline 99GG055 & 531250 & 7138700 & AMPH & 0.25 & & & & & & \\
\hline 99GG056 & 531375 & 7137700 & QFR & 0 & & & & & & \\
\hline 99GG057 & 531250 & 7138700 & GN & 0 & & & & & & \\
\hline 99GG058 & 531000 & 7137200 & QFR & 0 & & & & & & \\
\hline \begin{tabular}{|l|} 
99GG059 \\
\end{tabular} & 531375 & 7137700 & FGN & 0.03 & & & & & & \\
\hline 99GG060 & 532175 & 7138100 & QFR & 0 & & & & & & \\
\hline 99GG061 & 530300 & 7140550 & FGN & 0 & & & & & & \\
\hline 99GG062 & 530300 & 7140550 & QFR & 0 & & & & & & \\
\hline 99GG063 & 528450 & 7142660 & SKN & 0.2 & & & & & & \\
\hline 99GG064 & 531300 & 7137700 & RHY & 0 & & & & & & \\
\hline 99GG065 & 532150 & 7137300 & QFR & 0 & & & & & & \\
\hline 99GG066 & 532175 & 7138100 & SGN & 0.1 & & & & & & \\
\hline 99GG067 & 528270 & 7140760 & RHY & 0.01 & & & & & & \\
\hline 99GG068 & 535000 & 7137480 & MI & 0.06 & & & & & & \\
\hline 99GG069 & 536630 & 7137230 & MI & 0.09 & & & & & & \\
\hline 99GG070 & 536600 & 7137700 & MI & 0.08 & & & & & & \\
\hline 99GG071 & 536000 & 7137300 & QFR & 0 & & & & & & \\
\hline 99GG072 & 533910 & 7139100 & GN & 0.06 & $\mathrm{x}$ & & $\mathrm{x}$ & & & \\
\hline 99GG073 & 535270 & 7134050 & GN & 0.22 & & & & & & \\
\hline 99GG074 & 534900 & 7138450 & $\mathrm{GN}$ & 0.05 & & & & & & \\
\hline 99GG075 & 536100 & 7137000 & MI & 0.08 & & & & & & \\
\hline 99GG077 & 536000 & 7137750 & MTR & 0.05 & & & & & & \\
\hline 99GG078 & 536950 & 7136200 & QFR & 0 & & & & & & \\
\hline 99GG079 & 536100 & 7137150 & MTR & 0.03 & & & & & & \\
\hline 99GG080 & 534100 & 7139100 & QFR & 0 & & & & & & \\
\hline 99GG081 & 536100 & 7137150 & MI & 0.08 & & & & & & \\
\hline 99GG082 & 537200 & 7135700 & QFR & 0 & & & & & & \\
\hline 99GG083 & 537500 & 7135700 & MTR & 0.06 & & & & & & \\
\hline 99GG084 & 537100 & 7134700 & MTR & 0.08 & & & & & & \\
\hline 99GG085 & 534400 & 7133700 & SGN & 0.04 & & & & & & \\
\hline 99GG086 & 537200 & 7135700 & MTR & 0.08 & & & & & & \\
\hline 99GG087 & 537200 & 7135450 & ALT & 0 & & & & & & \\
\hline 99GG088 & 537130 & 7135730 & $\overline{B L G}$ & 0.4 & & & & & & \\
\hline 99GG089 & 538000 & 7135600 & $\overline{A L T}$ & 0 & & & & & & \\
\hline 99GG090 & 537300 & 7133650 & MTR & 0.09 & & & & & & \\
\hline 99GG091 & 535350 & 7133900 & QFR & 0 & & & & & & \\
\hline 99GG092 & 536000 & 7137650 & MI & 0.06 & & & & & & \\
\hline 99GG093 & 537200 & 7134900 & GN & 0.06 & & & & & & \\
\hline 99GG094 & 534100 & 7139100 & GN & 0.05 & $\mathrm{X}$ & & $\mathrm{x}$ & & & \\
\hline 99GG095 & 536150 & 7137100 & BLG & 1.3 & & & & & & \\
\hline 99GG096 & 536600 & 7137130 & QFR & 0 & & & & & & \\
\hline 99GG097 & 537200 & 7136800 & MI & 0.03 & & & & & & \\
\hline 99GG098 & 537750 & 7137400 & BLG & 0.15 & & & & & & \\
\hline 99GG099 & 537750 & 7137400 & ALT & 0 & & & & & & \\
\hline 99GG100 & 536200 & 7137000 & MI & 0.05 & & & & & & \\
\hline 99GG101 & 536630 & 7137230 & MI & 0.08 & & & & & & \\
\hline \begin{tabular}{|l|}
$99 G G 102$ \\
\end{tabular} & 536500 & 7135000 & MGN & 0.25 & & & & & & \\
\hline
\end{tabular}


Table 1 continued. List of grab samples, approximate locations, assigned rock types and analyses.

\begin{tabular}{|c|c|c|c|c|c|c|c|c|c|c|}
\hline Sample\# & UTMX & UTMY & Rock type & $\begin{array}{l}\text { Mag. Susc. } \\
\left(* 10^{\wedge}-3 \text { SI }\right)\end{array}$ & T.S. & P.S. & WRA & Geotherm & FI & Ar/Ar \\
\hline 99GG103 & 533850 & 7133250 & $\overline{G N}$ & 0.06 & & & & & & \\
\hline 99GG104 & 536140 & 7137090 & MI & 0 & & & & & & \\
\hline 99GG105 & 528200 & 7140050 & MI & 0.06 & $\mathrm{X}$ & & & & & \\
\hline 99GG106 & 527350 & 7139800 & MI & 0.08 & $\mathrm{x}$ & & & & & \\
\hline 99GG107 & 533850 & 7133090 & SKN & 0.03 & & & & & & \\
\hline 99GG108 & 530600 & 7139900 & GN & 0.1 & & & & & & \\
\hline 99GG109 & 530600 & 7139900 & QFR & 0.02 & & & & & & \\
\hline 99GG110 & 528000 & 7141300 & $\overline{\text { AGN }}$ & 0.02 & & & & & & \\
\hline 99GG111 & 536200 & 7137000 & BLG & 0.1 & & & & & & \\
\hline & & & & & & & & & & \\
\hline \multicolumn{11}{|c|}{ Drill hole samples and USGS samples } \\
\hline \begin{tabular}{|l|}
$77-187$ \\
(USGS)
\end{tabular} & 542000 & 7139000 & GN & & $\mathrm{x}$ & & & $\mathrm{x}$ & & \\
\hline $\begin{array}{l}77-452 \\
\text { (USGS) }\end{array}$ & 525000 & 7143000 & GN & & $\mathrm{x}$ & & & $\mathrm{x}$ & & \\
\hline $\begin{array}{l}77-576 \\
\text { (USGS) }\end{array}$ & 526000 & 7137200 & GN & & $\mathrm{x}$ & & & $\mathrm{x}$ & & \\
\hline BK1-035 & 536600 & 7136400 & BLG & 0.01 & & & & & $x$ & \\
\hline \begin{tabular}{|l|l|} 
BK1-082 \\
\end{tabular} & 536600 & 7136400 & BLG & 0.02 & & & & & $\mathrm{x}$ & \\
\hline BK1-65 & 536600 & 7136400 & BLG & 0.02 & & & & & $\mathrm{x}$ & \\
\hline \begin{tabular}{|l|} 
BK1-137 \\
\end{tabular} & 536600 & 7136400 & BLG & 0.05 & & $\mathrm{x}$ & & & & \\
\hline \begin{tabular}{|l} 
BK1-146 \\
\end{tabular} & 536600 & 7136400 & BLG & 0.52 & $\mathrm{x}$ & $\mathrm{x}$ & & & & \\
\hline \begin{tabular}{|l|} 
BK2-012 \\
\end{tabular} & 536830 & 7135800 & BLG & & & & & & $\bar{x}$ & \\
\hline \begin{tabular}{|l|} 
BK2-047 \\
\end{tabular} & 536830 & 7135800 & $\mathrm{BLG}+\mathrm{VN}$ & 0.05 & & & & & $\mathrm{x}$ & $\bar{x}$ \\
\hline BK2-65 & 536830 & 7135800 & BLG & $0.27-0.20$ & & $\mathrm{x}$ & & & & \\
\hline BK2-45 & 536830 & 7135800 & BLG & 0.05 & & $\mathrm{x}$ & & & & \\
\hline BK2-73 & 536830 & 7135800 & BLG & 0.21 & $\mathrm{x}$ & $\mathrm{x}$ & & & & \\
\hline BK2-98 & 536830 & 7135800 & BLG & 0.01 & & $\mathrm{x}$ & & & & \\
\hline \begin{tabular}{|l} 
BK2-131 \\
\end{tabular} & 536830 & 7135800 & BLG & 0.01 & & $\mathrm{x}$ & & & & \\
\hline \begin{tabular}{|l} 
BK2-135 \\
\end{tabular} & 536830 & 7135800 & BLG & 0 & & & & & $\mathrm{x}$ & \\
\hline \begin{tabular}{|l} 
BK2-152 \\
\end{tabular} & 536830 & 7135800 & BLG & 0.01 & & & & & $\mathrm{x}$ & \\
\hline \begin{tabular}{|l} 
BK3-021 \\
\end{tabular} & 536000 & 7135000 & BK dike 1 & 0 & $\mathrm{x}$ & & $\mathrm{x}$ & & & \\
\hline \begin{tabular}{|l|} 
BK3-130 \\
\end{tabular} & 536000 & 7135000 & BK dike 2 & 0.01 & $\mathrm{x}$ & & $\mathrm{x}$ & & & \\
\hline \begin{tabular}{|l|} 
BK3-142 \\
\end{tabular} & 536000 & 7135000 & GN & 0.04 & & & & $\mathrm{x}$ & & \\
\hline \begin{tabular}{|l|} 
BK4-062 \\
\end{tabular} & 536100 & 7136100 & BLG & 0.11 & $\bar{x}$ & & $\mathrm{X}$ & & & \\
\hline \begin{tabular}{|l|} 
BK4-263 \\
\end{tabular} & 536100 & 7136100 & BLG & 0.22 & $\mathrm{x}$ & & $\mathrm{x}$ & & & \\
\hline BK4-99 & 536100 & 7136100 & BLG & 0.08 & & $\mathrm{x}$ & & & & \\
\hline BK4-160 & 536100 & 7136100 & $\overline{B L G}$ & 0.17 & & $\bar{x}$ & & & & \\
\hline \begin{tabular}{|l|} 
BK4-146 \\
\end{tabular} & 536100 & 7136100 & BLG & 0.2 & & $\mathrm{x}$ & & & & \\
\hline BK5-65 & 536900 & 7135200 & BLG & 0.07 & & $\mathrm{x}$ & & & & \\
\hline BK5-98 & 536900 & 7135200 & BLG & $0.04-0.02$ & & $\mathrm{x}$ & & & & \\
\hline \begin{tabular}{|l|} 
BK5-148 \\
\end{tabular} & 536900 & 7135200 & BLG & 0.02 & & $\mathrm{x}$ & & & & \\
\hline BK6-59 & 536250 & 7135200 & BLG & 0.06 & & & $\mathrm{X}$ & & & \\
\hline \begin{tabular}{|l} 
BK5-150 \\
\end{tabular} & 536900 & 7135200 & BLG & 0.38 & $\mathrm{x}$ & $\mathrm{x}$ & $\mathrm{x}$ & & & \\
\hline \begin{tabular}{|l|} 
BK6-059 \\
\end{tabular} & 536250 & 7135200 & BLG & $0.06-0.14$ & $\mathrm{x}$ & $\mathrm{x}$ & $\mathrm{x}$ & & & \\
\hline BK6-121 & 536250 & 7135200 & $\begin{array}{l}\text { Migmatite } \\
\text { leucosome }\end{array}$ & 0 & & & $\mathrm{x}$ & & & \\
\hline \begin{tabular}{|l|} 
BK6-125 \\
\end{tabular} & 536250 & 7135200 & $\mathrm{GN}$ & 0.04 & & & $\mathrm{X}$ & & & \\
\hline
\end{tabular}


Table 1 continued. List of grab samples, approximate locations, assigned rock types and analyses.

\begin{tabular}{|c|c|c|c|c|c|c|c|c|c|c|}
\hline Sample\# & UTMX & UTMY & Rock type & $\begin{array}{l}\text { Mag. Susc. } \\
\left(* 10^{\wedge}-3 \text { SI }\right)\end{array}$ & T.S. & P.S. & WRA & Geotherm & FI & Ar/Ar \\
\hline BK6-129 & 536250 & 7135200 & $\begin{array}{l}\text { Migmatite } \\
\text { leucosome }\end{array}$ & 0.01 & & & $\mathrm{x}$ & & & \\
\hline BK6-131 & 536250 & 7135200 & \begin{tabular}{|l|} 
Migmatite \\
leucosome \\
\end{tabular} & 0.01 & & & $\mathrm{x}$ & & & \\
\hline \begin{tabular}{|l|} 
BR1-372 \\
\end{tabular} & SW Buck & & GN & & $\mathrm{X}$ & & $\mathrm{x}$ & & & \\
\hline \begin{tabular}{|l|} 
BR4-323 \\
\end{tabular} & SW Buck & & GN & & $\bar{x}$ & & $\bar{x}$ & & & \\
\hline \begin{tabular}{|l|} 
BR4-328 \\
\end{tabular} & SW Buck & & $\mathrm{GN}+\mathrm{VN}$ & & $\mathrm{x}$ & & & & $\mathrm{x}$ & $\mathrm{X}$ \\
\hline \begin{tabular}{|l|} 
BR4-334 \\
\end{tabular} & SW Buck & & GN & & $\bar{x}$ & & $\bar{x}$ & & & \\
\hline \begin{tabular}{|l|} 
Buckeye \\
Discovery
\end{tabular} & 536330 & 7134900 & $\begin{array}{l}\text { Quartz, GN, } \\
\text { PEG, AGN }\end{array}$ & & $\mathrm{x}$ & & & & $\mathrm{x}$ & \\
\hline GRI-1 & 528200 & 7141500 & $\begin{array}{c}\text { Gold Run } \\
\text { Intrusion }\end{array}$ & 0.01 & $\mathrm{x}$ & & $\mathrm{x}$ & & & $\mathrm{x}$ \\
\hline GRI-2 & 528630 & 7141175 & $\begin{array}{c}\text { Gold Run } \\
\text { Intrusion }\end{array}$ & 0.01 & $\mathrm{x}$ & & $\mathrm{x}$ & & & \\
\hline GRI-3 & 528400 & 7141350 & $\begin{array}{l}\text { Gold Run } \\
\text { Intrusion }\end{array}$ & 0.01 & $\mathrm{x}$ & & $\mathrm{x}$ & & & \\
\hline
\end{tabular}

\section{Legend:}

MTR, MI, BLG = Bald knob gneiss

GN = undifferentiated gneiss

$\mathrm{SKN}=$ marble

$\mathrm{QFR}=$ felsic dike

$\mathrm{SCH}=$ schistose rock

SGN = schistose gneiss (undifferentiated)

HFL $=$ hornfels

$\mathrm{QFT}=$ tourmaline bearing felsic gneiss

ALT $=$ bleached gneissic rock (predominately on Buck Ridge)

RHY = rhyolite

AGN $=$ augen gneiss

PEG = pegmatite

AMPH = amphibolite

QTZ = quartz-rich rock 
Table 2. Modal mineralogy of gneisses. Values are visual estimates of percentages from petrographic analysis. UTMX and UTMY are UTM coordinates. Plag=plagioclase, Kspar= K-feldspar, Biot=biotite, Sill=sillimanite, WM=white mica,

Gar=garnet, $\mathrm{Chl}=$ chlorite, Tr=trace. Buck Ridge coordinates are not known.

\begin{tabular}{|c|c|c|c|c|c|c|c|c|c|c|c|}
\hline Sample & UTMX & UTMY & Quartz & Plag & Kspar & Biot & Opaques & Sill & $\mathbf{W M}$ & Gar & Chl \\
\hline 99GG-24 & 528460 & 7142640 & 27 & 26 & & 20 & 5 & 15 & 12 & & \\
\hline 122B & 530250 & 7139050 & 60 & 20 & & 10 & 2 & 4 & 4 & & \\
\hline 99GG-106 & 527350 & 7139800 & 50 & 15 & 10 & 18 & & 5 & 2 & & \\
\hline 99GG-105 & 528200 & 7140050 & 45 & 10 & 19 & 20 & & 5 & & 1 & \\
\hline 99GG-43 & 530500 & 7140000 & 48 & 10 & 10 & 20 & 1 & 10 & 1 & & \\
\hline 99GG-40 & 529050 & 7139300 & 60 & 25 & & 14 & 1 & & & & \\
\hline 99GG-31 & 528490 & 7140274 & 60 & 20 & & 15 & 1 & 10 & & & \\
\hline 99GG-26 & 528350 & 7140000 & 50 & 17 & 7 & 15 & & 5 & & 1 & \\
\hline 99GG-13 & 527350 & 7139800 & 45 & 14 & 5 & 25 & 10 & & & 1 & \\
\hline 99GG-08 & 528200 & 7140040 & 50 & 17 & 5 & 20 & & 5 & 2 & 1 & \\
\hline 122A & 530250 & 7139050 & 39 & 35 & & 20 & 5 & & & 1 & \\
\hline 99GG-09 & 527840 & 7140035 & 55 & 10 & & 20 & & 5 & 10 & & \\
\hline 118A & 528650 & 7138950 & 50 & 25 & & 13 & & 1 & 10 & 1 & \\
\hline 99GG-18 & 528410 & 7141750 & 50 & 15 & & 10 & 2 & 2 & 15 & & 6 \\
\hline 123 & 530500 & 7139050 & 65 & 15 & & 15 & & 4 & & 1 & \\
\hline 128 & 530860 & 7138960 & 30 & 30 & & 19 & & 10 & 10 & & 1 \\
\hline 124B & 530650 & 7139000 & 60 & 20 & & 15 & & & 5 & & \\
\hline 124C & 530650 & 7139000 & 60 & 20 & & 10 & & 2 & 10 & & \\
\hline 137 & 531750 & 7140500 & 60 & 15 & & 20 & & 5 & & & \\
\hline 81 & 534800 & 7134250 & 62 & 22 & & 15 & & & 1 & & \\
\hline 142 & 533000 & 7140400 & 65 & 18 & & 10 & & 1 & 5 & 1 & \\
\hline 140 & 532900 & 7140450 & 54 & 23 & & 15 & & 3 & 5 & & \\
\hline 99GG-094 & 534100 & 7139100 & 30 & 45 & 10 & 14 & & & & & 1 \\
\hline 99GG-072 & 533910 & 7139100 & 25 & 40 & 20 & 13 & & & & 1 & 1 \\
\hline 122a & 530250 & 7139050 & 40 & 40 & & 19 & & & & 1 & \\
\hline 77-187 & 542000 & 7139000 & 50 & 25 & 5 & 15 & & 2 & 2 & 1 & \\
\hline $77 W-452$ & 525000 & 7143000 & 50 & 30 & & 20 & & & & 1 & \\
\hline BK3-142 & 536000 & 7135000 & 45 & 25 & 5 & 20 & & 4 & & 1 & \\
\hline $77-576$ & 526000 & 7137200 & 50 & 28 & 5 & 15 & & 2 & & 1 & \\
\hline BK6-059 & 536250 & 7135200 & 40 & 20 & 15 & 17 & 2 & 1 & 5 & & \\
\hline BK5-150 & 536900 & 7135200 & 45 & 20 & 7 & 15 & 3 & 3 & 7 & & \\
\hline BK4-062 & 536100 & 7136100 & 50 & 25 & 5 & 15 & 1 & 5 & 5 & & \\
\hline BK4-263 & 536100 & 7136100 & 40 & 20 & 15 & 15 & 2 & 1 & 5 & & \\
\hline BR4-323 & \multicolumn{2}{|c|}{ SW Buck } & 55 & 20 & & 24 & & 1 & & & \\
\hline \begin{tabular}{|l|} 
BR1-372 \\
\end{tabular} & \multicolumn{2}{|c|}{ SW Buck } & 50 & 25 & & 20 & 2 & 3 & & & \\
\hline BR4-334 & \multicolumn{2}{|c|}{ SW Buck } & 55 & 15 & 15 & 15 & & Tr. & & & \\
\hline
\end{tabular}

Table 3. Estimated modal mineral abundances (volume \%) in felsic dike thin sections.

\begin{tabular}{|c|c|c|c|c|c|c|c|}
\hline Sample & Group & Qtz & Plag & Kspar & Tourm & Mica & Point Cnts. \\
\hline 73 & 1 & 36 & 30 & 32 & 2 & & 102 \\
\hline 143a & 1 & 35 & 27 & 34 & & 4 & 104 \\
\hline 125 & 1 & 33 & 27 & 32 & & 8 & 109 \\
\hline 158a & 2 & 36 & 34 & 26 & & $4^{*}$ & 104 \\
\hline
\end{tabular}

Qtz=quartz, Plag=plagioclase, Kspar=K-feldspar, Tourm=tourmaline, Mica=white mica and minor biotite, Point Cnts.= number of points counted, *=biotite altering to chlorite. 
Table 4. XRF major oxide and normative values for gneiss samples in the Richardson study area. An=anorthite, Q=quartz, or=orthoclase, $\mathrm{ab}=$ albite, $\mathrm{C}=$ =corundum, hy=hypersthene, $\mathrm{mt}=$ magnetite, il=ilmenite, hem=hematite, ap=apatite.

\begin{tabular}{|c|c|c|c|c|c|c|c|c|c|c|c|}
\hline Sample & 118A & 112A & 123 & 128 & 124B & $124 \mathrm{C}$ & 137 & 140 & 142 & 81 & 99GG094 \\
\hline $\mathrm{SiO} 2$ & 71.4 & 81.28 & 85.4 & 60.18 & 83.66 & 73.76 & 65.98 & 69.93 & 84.07 & 78.42 & 71.81 \\
\hline TiO2 & 0.55 & 0.31 & 0.25 & 0.77 & 0.24 & 0.53 & 0.67 & 0.68 & 0.18 & 0.4 & 0.28 \\
\hline Al2O3 & 13.77 & 9.23 & 6.34 & 20.34 & 7.39 & 12.05 & 16.75 & 14.99 & 7.29 & 9.59 & 14.36 \\
\hline $\mathrm{Fe} 2 \mathrm{O} 3$ & 3.94 & 2.54 & 2.13 & 6.71 & 2.25 & 4.88 & 5.21 & 5.87 & 2.55 & 3.46 & 3.08 \\
\hline MnO & 0.06 & 0.04 & 0.04 & 0.07 & 0.03 & 0.05 & 0.04 & 0.09 & 0.03 & 0.04 & 0.07 \\
\hline MgO & 1.35 & 0.78 & 0.5 & 2.27 & 0.53 & 1.59 & 1.82 & 1.77 & 0.64 & 1.25 & 0.7 \\
\hline $\mathrm{CaO}$ & 2.68 & 0.56 & 0.57 & 0.56 & 0.71 & 0.56 & 0.46 & 0.61 & 0.92 & 1.11 & 2.53 \\
\hline $\mathrm{Na} 2 \mathrm{O}$ & 3.22 & 1.13 & 1.08 & 1.23 & 1.54 & 1.23 & 1.24 & 0.63 & 1.49 & 1.7 & 3.08 \\
\hline K2O & 1.35 & 1.91 & 0.99 & 3.92 & 1.33 & 2.82 & 3.84 & 2.91 & 1.13 & 2.5 & 2.75 \\
\hline P2O5 & 0.05 & 0.07 & 0.05 & 0.09 & 0.04 & 0.07 & 0.08 & 0.09 & 0.08 & 0.08 & 0.09 \\
\hline LOI & 0.8 & 1.16 & 0.76 & 3.45 & 0.81 & 1.42 & 2.72 & 1.85 & 0.79 & 0.75 & 0.84 \\
\hline Total & 99.2 & 99.02 & 98.14 & 99.61 & 98.56 & 99 & 98.82 & 99.44 & 99.21 & 99.33 & 99.62 \\
\hline$\%$ AN & 32.25 & 19.53 & 21.49 & 17.38 & 20.02 & 18.23 & 14.36 & 31.38 & 24.27 & 25.73 & 31.46 \\
\hline $\mathbf{Q}$ & 39.61 & 65.24 & 73.51 & 31.73 & 67.42 & 51.42 & 39.6 & 49.92 & 68.38 & 54.76 & 37.01 \\
\hline or & 7.98 & 11.29 & 5.85 & 23.17 & 7.86 & 16.67 & 22.69 & 17.2 & 6.68 & 14.77 & 16.25 \\
\hline $\mathbf{a b}$ & 27.25 & 9.56 & 9.14 & 10.41 & 13.03 & 10.41 & 10.49 & 5.33 & 12.61 & 14.39 & 26.06 \\
\hline an & 12.97 & 2.32 & 2.5 & 2.19 & 3.26 & 2.32 & 1.76 & 2.44 & 4.04 & 4.98 & 11.96 \\
\hline C & 2.26 & 4.45 & 2.58 & 13.27 & 2.22 & 6.12 & 9.91 & 9.91 & 2.13 & 2.26 & 1.93 \\
\hline hy & 4 & 1.94 & 1.25 & 9.97 & 1.32 & 6.21 & 6.73 & 7.75 & 1.59 & 3.54 & 2.09 \\
\hline mt & 2.97 & 1.35 & 0.51 & 3.29 & 0.88 & 2.94 & 3.15 & 3.16 & 2.1 & 2.75 & 2.58 \\
\hline il & 1.04 & 0.59 & 0.47 & 1.46 & 0.46 & 1.01 & 1.27 & 1.29 & 0.34 & 0.76 & 0.53 \\
\hline hem & 0 & 0.88 & 1.4 & 0 & 1.13 & 0 & 0 & 0 & 0.23 & 0 & 0 \\
\hline ap & 0.12 & 0.16 & 0.12 & 0.21 & 0.09 & 0.16 & 0.19 & 0.21 & 0.19 & 0.19 & 0.21 \\
\hline
\end{tabular}


Table 4 continued. XRF major oxide and normative values for gneiss samples in the Richardson study area.

\begin{tabular}{|c|c|c|c|c|c|c|c|c|c|c|c|}
\hline Sample & 99GG072 & 99GG024 & 99GG031 & 99GG040 & BK004-062 & BK004-263 & BK005-150 & BK006-059 & BR1-372 & BR4-323 & BR4-334 \\
\hline $\mathrm{SiO} 2$ & 70.92 & 55.15 & 73.96 & 85.21 & 69.13 & 69.92 & 70.5 & 69.56 & 73.64 & 71.4 & 71.51 \\
\hline TiO2 & 0.27 & 1.13 & 0.58 & 0.24 & 0.77 & 0.84 & 0.79 & 0.57 & 0.53 & 0.62 & 0.58 \\
\hline Al2O3 & 14.5 & 23.92 & 12.82 & 6.44 & 14.46 & 14 & 14.68 & 14.05 & 13.23 & 14.69 & 14.08 \\
\hline Fe2O3 & 2.95 & 7.84 & 4.84 & 2.28 & 5.55 & 5.09 & 6.5 & 4.64 & 4.52 & 5.37 & 4.37 \\
\hline MnO & 0.06 & 0.15 & 0.07 & 0.05 & 0.04 & 0.05 & 0.07 & 0.06 & 0.07 & 0.06 & 0.05 \\
\hline MgO & 0.64 & 2.86 & 1.49 & 0.52 & 1.74 & 1.55 & 1.98 & 1.51 & 1.35 & 1.69 & 1.47 \\
\hline $\mathrm{CaO}$ & 2.62 & 0.76 & 0.81 & 0.76 & 1.39 & 1.24 & 0.6 & 1.13 & 0.87 & 0.67 & 0.83 \\
\hline $\mathrm{Na} 2 \mathrm{O}$ & 2.64 & 1.47 & 1.19 & 1.54 & 1.3 & 1.34 & 0.75 & 1.62 & 0.97 & 0.9 & 1.21 \\
\hline K2O & 3.84 & 3.98 & 2.33 & 0.85 & 3.46 & 3.8 & 2.12 & 3.48 & 2.57 & 2.96 & 3.41 \\
\hline P2O5 & 0.09 & 0.09 & 0.08 & 0.06 & 0.09 & 0.1 & 0.08 & 0.1 & 0.09 & 0.09 & 0.11 \\
\hline LOI & 0.66 & 1.95 & 1.28 & 0.74 & 1.46 & 1.2 & 1.72 & 1.45 & 1.86 & 1.64 & 1.07 \\
\hline Total & 99.21 & 99.33 & 99.48 & 98.72 & 99.39 & 99.13 & 99.79 & 98.17 & 99.7 & 100.09 & 98.69 \\
\hline$\% \mathrm{AN}$ & 35.71 & 20.37 & 25.77 & 20.59 & 36.44 & 32.66 & 27.88 & 26.54 & 31.23 & 26.43 & 24.92 \\
\hline $\mathbf{Q}$ & 34.49 & 23.52 & 53.48 & 70.77 & 41.94 & 42.3 & 52.25 & 41.66 & 53.77 & 49.91 & 47.22 \\
\hline or & 22.69 & 23.52 & 13.77 & 5.02 & 20.45 & 22.46 & 12.53 & 20.57 & 15.19 & 17.49 & 20.15 \\
\hline ab & 22.34 & 12.44 & 10.07 & 13.03 & 11 & 11.34 & 6.35 & 13.71 & 8.21 & 7.62 & 10.24 \\
\hline an & 12.41 & 3.18 & 3.5 & 3.38 & 6.31 & 5.5 & 2.45 & 4.95 & 3.73 & 2.74 & 3.4 \\
\hline C & 1.45 & 16.03 & 7.06 & 1.75 & 6.26 & 5.67 & 10.25 & 5.8 & 7.49 & 9 & 7.15 \\
\hline hy & 1.75 & 11.97 & 5.73 & 1.3 & 6.68 & 5.18 & 8.82 & 5.47 & 5.05 & 6.92 & 4.86 \\
\hline mt & 2.57 & 3.81 & 3.02 & 1.03 & 3.29 & 3.39 & 3.32 & 3 & 2.94 & 3.07 & 3.02 \\
\hline il & 0.51 & 2.15 & 1.1 & 0.46 & 1.46 & 1.6 & 1.5 & 1.08 & 1.01 & 1.18 & 1.1 \\
\hline hem & 0 & 0 & 0 & 1.03 & 0 & 0 & 0 & 0 & 0 & 0 & 0 \\
\hline ap & 0.21 & 0.21 & 0.19 & 0.14 & 0.21 & 0.23 & 0.19 & 0.23 & 0.21 & 0.21 & 0.25 \\
\hline
\end{tabular}


Table 5. XRF major and minor element results for amphibolite samples 127 and 158B. Oxides are shown in percent and trace elements are in ppm.

\begin{tabular}{|ccc|}
\hline Samples & $\mathbf{1 2 7}$ & $\mathbf{1 5 8 B}$ \\
\hline SiO2 & 44.87 & 39.4 \\
TiO2 & 4.05 & 5.77 \\
Al2O3 & 5.94 & 10.84 \\
Fe2O3 & 13.3 & 15.04 \\
MnO & 0.29 & 0.28 \\
MgO & 15.74 & 8.02 \\
CaO & 11.57 & 13.41 \\
Na2O & 0.6 & 0.5 \\
K2O & 0.17 & 0.75 \\
P2O5 & 0.77 & 2.22 \\
LOI & 1.61 & 3.14 \\
Total & 99.06 & 99.46 \\
\hline Ba & 228 & 119 \\
Sr & 159 & 164 \\
$\mathbf{Y}$ & 18 & 34 \\
Nb & 65 & 92 \\
Zr & 241 & 336 \\
Rb & 12 & 21 \\
\hline
\end{tabular}


Table 6. XRF major oxide and normative values in percent (\%) for igneous rock samples in the Richardson study area. Norm abbreviations are the same as for table 4. *Sample BK3-125 shown for comparison to adjacent migmatite leucosomes.

\begin{tabular}{|c|c|c|c|c|c|c|c|c|}
\hline Sample & 73 & 125 & 158 & 129 & 135 & 143 & BK003-021 & BK003-130 \\
\hline Rock type & Felsic dike & Felsic dike & Felsic dike & Felsic dike & Felsic dike & Felsic dike & Course dike & Course dike \\
\hline $\mathrm{SiO} 2$ & 74.97 & 74.93 & 74.67 & 72.39 & 74.64 & 73.75 & 73.18 & 69.21 \\
\hline TiO2 & 0.03 & 0.08 & 0.09 & 0.08 & 0.05 & 0.04 & 0.03 & 0.14 \\
\hline Al2O3 & 14.23 & 14.18 & 14.34 & 13.94 & 14.4 & 14.22 & 15.59 & 15.98 \\
\hline Fe2O3 & 0.89 & 0.61 & 0.72 & 1.21 & 0.6 & 0.96 & 0.43 & 1.15 \\
\hline MnO & 0.08 & 0.02 & 0.01 & 0.01 & 0 & 0.02 & 0 & 0.02 \\
\hline MgO & 0.11 & 0.22 & 0.28 & 0.19 & 0.11 & 0.09 & 0.14 & 0.28 \\
\hline $\mathrm{CaO}$ & 0.42 & 0.52 & 1.38 & 0.46 & 0.46 & 0.67 & 2.15 & 1.57 \\
\hline $\mathrm{Na2O}$ & 3.4 & 2.88 & 3.79 & 2.78 & 2.45 & 3.35 & 3.23 & 2.34 \\
\hline K2O & 4.17 & 4.99 & 3.39 & 4.3 & 4.43 & 4.34 & 5.09 & 7.6 \\
\hline P2O5 & 0.17 & 0.14 & 0.1 & 0.1 & 0.06 & 0.2 & 0.07 & 0.54 \\
\hline LOI & 0.79 & 0.85 & 0.75 & 2.54 & 1.74 & 1.08 & 0.4 & 0.71 \\
\hline Total & 99.26 & 99.42 & 99.52 & 98 & 98.94 & 98.72 & 100.31 & 99.54 \\
\hline$\% A N$ & 3.27 & 6.4 & 16.18 & 6.48 & 8.36 & 6.64 & 27.19 & 17.71 \\
\hline $\mathbf{Q}$ & 38.65 & 38.04 & 36.56 & 38.78 & 42.46 & 36.65 & 30.3 & 24.26 \\
\hline or & 24.64 & 29.49 & 20.03 & 25.41 & 26.18 & 25.65 & 30.08 & 44.91 \\
\hline $\mathbf{a b}$ & 28.77 & 24.37 & 32.07 & 23.52 & 20.73 & 28.35 & 27.33 & 19.8 \\
\hline an & 0.97 & 1.67 & 6.19 & 1.63 & 1.89 & 2.02 & 10.21 & 4.26 \\
\hline C & 3.77 & 3.43 & 2.17 & 4.12 & 4.88 & 3.27 & 1.03 & 2.34 \\
\hline hy & 0.27 & 0.55 & 0.7 & 0.47 & 0.27 & 0.22 & 0.35 & 0.7 \\
\hline mt & 0.17 & 0 & 0 & 0 & 0 & 0 & -0.02 & 0.04 \\
\hline il & 0.06 & 0.04 & 0.02 & 0 & 0 & 0 & 0.43 & 1.15 \\
\hline hem & 0.77 & 0.61 & 0.72 & 1.21 & 0.6 & 0.96 & 0.16 & 1.25 \\
\hline ap & 0.39 & 0.32 & 0.23 & 0.23 & 0.14 & 0.46 & 0.04 & 0.12 \\
\hline ru & 0 & 0.06 & 0.08 & 0.1 & 0.07 & 0.04 & 0 & 0 \\
\hline
\end{tabular}


Table 6 continued. XRF major oxide and normative values in percent (\%) for igneous rock samples in the Richardson study area.

\begin{tabular}{|c|c|c|c|c|c|c|c|}
\hline Sample & GRI-1 & GRI-2 & GRI-3 & BK6-128 & BK6-131 & BK6-121 & BK3-125 \\
\hline Rock type & Gold Run & Gold Run & Gold Run & Migmatite & Migmatite & Migmatite & Gneiss* \\
\hline $\mathrm{SiO} 2$ & 71.4 & 72 & 71.54 & 73.01 & 74.01 & 73.01 & 71.07 \\
\hline TiO2 & 0.28 & 0.28 & 0.25 & 0.03 & 0.14 & 0.09 & 0.6 \\
\hline Al2O3 & 14.69 & 14.27 & 14.36 & 14.84 & 13.96 & 14.67 & 13.08 \\
\hline Fe2O3 & 2.66 & 2.28 & 2.6 & 0.87 & 0.95 & 1.05 & 4.3 \\
\hline MnO & 0.05 & 0.04 & 0.04 & 0.02 & 0 & 0.01 & 0.07 \\
\hline MgO & 0.66 & 0.6 & 0.59 & 0.4 & 0.41 & 0.42 & 1.47 \\
\hline $\mathrm{CaO}$ & 2.2 & 1.85 & 1.92 & 1.15 & 0.9 & 0.81 & 1.2 \\
\hline $\mathrm{Na} 2 \mathrm{O}$ & 3.06 & 3.07 & 2.98 & 1.86 & 1.79 & 1.91 & 1.89 \\
\hline K2O & 4.57 & 4.49 & 4.67 & 6.04 & 5.91 & 6.19 & 4.7 \\
\hline P2O5 & 0.11 & 0.15 & 0.11 & 0.23 & 0.08 & 0.08 & 0.1 \\
\hline LOI & 0.48 & 0.53 & 0.71 & 1.31 & 1.52 & 1.18 & 1.01 \\
\hline Total & 100.16 & 99.56 & 99.77 & 99.76 & 99.68 & 99.42 & 99.52 \\
\hline \%AN & 28.25 & 23.99 & 25.88 & 21.07 & 20.65 & 17.78 & 24.89 \\
\hline $\mathbf{Q}$ & 30.73 & 32.53 & 31.65 & 36.67 & 38.67 & 36.08 & 37.14 \\
\hline or & 27.01 & 26.53 & 27.6 & 35.69 & 34.93 & 36.58 & 27.78 \\
\hline ab & 25.89 & 25.98 & 25.22 & 15.74 & 15.15 & 16.16 & 15.99 \\
\hline an & 10.2 & 8.2 & 8.81 & 4.2 & 3.94 & 3.5 & 5.3 \\
\hline C & 0.97 & 1.36 & 1.18 & 3.7 & 3.17 & 3.55 & 2.94 \\
\hline hy & 1.64 & 1.49 & 1.47 & 1 & 1.02 & 1.05 & 4.7 \\
\hline $\mathrm{mt}$ & 1.9 & 0.77 & 1.87 & 0 & 0 & 0 & 3.04 \\
\hline il & 0.53 & 0.53 & 0.47 & 0.04 & 0 & 0.02 & 1.14 \\
\hline hem & 0.47 & 1.25 & 0.46 & 0.87 & 0.95 & 1.05 & 0 \\
\hline ap & 0.25 & 0.35 & 0.25 & 0.53 & 0.19 & 0.19 & 0.23 \\
\hline ru & 0 & 0 & 0 & 0.01 & 0.15 & 0.08 & 0 \\
\hline
\end{tabular}


Table 7. Trace element compositions (in ppm) of igneous rocks from the Richardson study area.

\begin{tabular}{|c|c|c|c|c|c|c|c|c|}
\hline Sample & Unit & Ba & Nb & Rb & Sr & $\mathbf{Y}$ & Zr & Nb+Y \\
\hline GRI-3 & Gold Run Intrusion & 1319 & 15 & 204 & 329 & 24 & 146 & 39 \\
\hline GRI-1 & Gold Run Intrusion & 1194 & 15 & 201 & 311 & 25 & 143 & 40 \\
\hline GRI-2 & Gold Run Intrusion & 1200 & 14 & 203 & 314 & 22 & 145 & 36 \\
\hline BK3-021 & BK dike 1 & 1284 & 3 & 103 & 299 & 10 & 62 & 13 \\
\hline BK3-130 & BK dike 2 & 2013 & 6 & 184 & 364 & 31 & 29 & 37 \\
\hline $\mathbf{1 2 5}$ & Felsic dike & 487 & 5 & 113 & 136 & 15 & 41 & 20 \\
\hline $\mathbf{1 5 8}$ & Felsic dike & 1763 & 5 & 68 & 402 & 14 & 34 & 19 \\
\hline $\mathbf{7 3}$ & Felsic dike & 236 & 30 & 298 & 33 & 18 & 28 & 48 \\
\hline $\mathbf{1 2 9}$ & Altered felsic dike & 372 & 12 & 133 & 133 & 21 & 47 & 33 \\
\hline $\mathbf{1 3 5}$ & Altered felsic dike & 239 & 22 & 159 & 119 & 19 & 44 & 41 \\
\hline $\mathbf{1 4 3}$ & Felsic dike & 201 & 25 & 263 & 53 & 17 & 39 & 42 \\
\hline BK6-121 & Migmatite leucosome & 1824 & 3 & 140 & 313 & 15 & 102 & 18 \\
\hline BK6-129 & Migmatite leucosome & 1630 & 1 & 134 & 299 & 16 & 56 & 17 \\
\hline BK6-131 & Migmatite leucosome & 1470 & 5 & 139 & 273 & 23 & 56 & 28 \\
\hline
\end{tabular}

Table 8. Samples employed for Geothermometry and Geobarometry from Richardson field area.

\begin{tabular}{|c|c|c|c|l|c|c|}
\hline Sample & UTMY & UTMX & $\begin{array}{c}\text { Fault } \\
\text { Block }\end{array}$ & Mineral assemblage present & $\begin{array}{c}\text { Geobarometer(s) } \\
\text { employed }\end{array}$ & Temp Est. $\left({ }^{\mathbf{0}} \mathbf{C}\right)$ \\
\hline $\mathbf{7 7 - 1 8 7}$ & 7139000 & 542000 & NE & Quartz-plagioclase-bio-musc-sillimanite-garnet & $1,2,3,4,5$ & 610,555 \\
\hline BK3-142 & 7135000 & 536000 & E & Quartz-plagioclase-biotite-sillimanite-garnet & 1 & 620 \\
\hline $\mathbf{1 2 2 a}$ & 7139050 & 530250 & W & Quartz-plagioclase-biotite-garnet & 1 & 5 \\
\hline $\mathbf{7 7 - 5 7 6}$ & 7137200 & 526000 & S & Quartz-plagioclase-biotite-sillimanite-garnet & 1 & \\
\hline $\mathbf{7 7 - 4 5 2}$ & 7143000 & 525000 & NW & Quartz-plagioclase-biotite-garnet & 2,3 & 555 \\
\hline
\end{tabular}

Geobarometers: 1=garnet-sillimanite-plagioclase-quartz (Hodges and Spear, 1982; Hodges and Crowley, 1985; Newton and Haselton, 1981;

Ganguly and Saxena, 1984), 2=garnet-muscovite-plagioclase-biotite (Hodges and Crowley, 1985; Ghent and Stout, 1981; Hoisch, 1990), 3=garnetmuscovite-plagioclase-quartz (Hoisch, 1990), 4=garnet-muscovite-sillimanite-quartz (Hodges and Crowley, 1985), 5=garnet-muscovite-biotitesillimanite (Hodges and Crowley, 1985). 
Table 9. Average microprobe data of mineral assemblages for geothermobarometric calculations.

\begin{tabular}{|c|c|c|c|c|c|c|c|c|c|c|c|c|c|}
\hline sample & $452 c-1$ & $452 c-1$ & $452 c-2$ & $452 c-2$ & $452 c-2$ & $452 \mathrm{c}-2$ & 77187-1 & 77187-1 & \begin{tabular}{|l|l|}
$77187-1$ \\
\end{tabular} & \begin{tabular}{|l|l|}
$77187-2$ \\
\end{tabular} & \begin{tabular}{|l|l|}
$77187-2$ \\
\end{tabular} & \begin{tabular}{|l|l|}
$77187-2$ \\
\end{tabular} & \begin{tabular}{|l|l|}
$77187-2$ \\
\end{tabular} \\
\hline mineral & g & b & g & h & wm & p & g & $\mathbf{p}$ & b & $\mathbf{p}$ & g & b & wm \\
\hline $\mathrm{Na} 2 \mathrm{O}$ & 0.05 & 0.11 & 0.03 & \begin{tabular}{l|l|}
0.07 \\
\end{tabular} & 1.01 & 9.49 & 0.05 & 7.94 & 0.13 & 7.78 & 0.02 & 0.14 & 0.61 \\
\hline MgO & 2.46 & 8.77 & 2.57 & 7.90 & 0.51 & 0.00 & 1.90 & 0.01 & 6.20 & 0.00 & 1.55 & 6.74 & 0.54 \\
\hline Al2O3 & 21.74 & 19.71 & 22.02 & 20.68 & 36.39 & 23.29 & 21.70 & 24.87 & 21.12 & 25.57 & 21.66 & 21.61 & 37.39 \\
\hline SiO2 & 36.83 & 34.26 & 37.07 & 35.23 & 43.62 & 63.85 & 36.80 & 61.26 & 33.93 & 60.23 & 36.79 & 34.53 & 45.08 \\
\hline $\mathrm{K} 2 \mathrm{O}$ & 0.05 & 8.91 & 0.01 & 8.36 & 9.58 & 0.15 & 0.01 & 0.35 & 8.76 & 0.25 & 0.02 & 8.84 & 10.32 \\
\hline $\mathrm{CaO}$ & 1.19 & 0.05 & 1.30 & \begin{tabular}{l|l}
0.09 \\
\end{tabular} & \begin{tabular}{l|l|}
0.02 \\
\end{tabular} & 3.33 & 1.62 & 5.37 & 0.05 & 5.98 & 1.49 & 0.04 & 0.05 \\
\hline TiO2 & 0.04 & 1.75 & 0.05 & 1.10 & 0.31 & 0.07 & 0.02 & 0.01 & 1.67 & 0.07 & 0.00 & 1.40 & 0.19 \\
\hline $\mathrm{FeO}$ & 32.06 & 19.30 & 33.49 & 19.90 & 1.18 & 0.01 & 30.37 & 0.03 & 21.38 & 0.06 & 28.57 & 20.26 & 1.12 \\
\hline $\mathrm{MnO}$ & 6.09 & 0.18 & 4.20 & 0.16 & 0.04 & 0.02 & 8.43 & 0.01 & 0.42 & 0.01 & 10.32 & 0.48 & 0.08 \\
\hline H2Oc & & 3.85 & & 3.89 & 4.38 & & & & 3.84 & & & 3.88 & 4.51 \\
\hline Total & 100.50 & 96.89 & 100.72 & 97.36 & 97.05 & 100.21 & 100.89 & 99.84 & 97.50 & 99.95 & 100.43 & 97.91 & 99.90 \\
\hline \multicolumn{14}{|c|}{$\begin{array}{l}\text { cations per } \\
\text { formula unit }\end{array}$} \\
\hline $\mathrm{Na}$ & 0.01 & 0.03 & 0.00 & 0.02 & 0.27 & 1.62 & 0.01 & 1.37 & 0.04 & 1.34 & 0.00 & 0.04 & 0.16 \\
\hline Mg & 0.30 & 2.04 & 0.31 & 1.82 & 0.10 & 0.00 & 0.23 & 0.00 & 1.44 & 0.00 & 0.19 & 1.55 & 0.11 \\
\hline Al & 2.06 & 3.62 & 2.08 & 3.76 & 5.86 & 2.41 & 2.06 & 2.60 & 3.89 & 2.68 & 2.06 & 3.93 & 5.85 \\
\hline Si & 2.96 & 5.34 & 2.97 & 5.43 & 5.96 & 5.62 & 2.96 & 5.44 & 5.29 & 5.36 & 2.97 & 5.33 & 5.99 \\
\hline $\mathbf{K}$ & 0.00 & 1.77 & 0.00 & 1.64 & 1.67 & 0.02 & 0.00 & 0.04 & 1.74 & 0.03 & 0.00 & 1.74 & 1.75 \\
\hline Ca & 0.10 & 0.01 & 0.11 & 0.01 & 0.00 & 0.31 & 0.14 & 0.51 & 0.01 & 0.57 & 0.13 & 0.01 & 0.01 \\
\hline $\mathrm{Ti}$ & 0.00 & 0.20 & 0.00 & 0.13 & 0.03 & 0.00 & 0.00 & 0.00 & 0.20 & 0.00 & 0.00 & 0.16 & 0.02 \\
\hline $\mathbf{F e}$ & 2.16 & 2.51 & 2.24 & 2.57 & 0.14 & 0.00 & 2.04 & 0.00 & 2.79 & 0.00 & 1.93 & 2.61 & 0.12 \\
\hline Mn & 0.41 & 0.02 & 0.28 & 0.02 & 0.00 & 0.00 & 0.57 & 0.00 & 0.06 & 0.00 & 0.71 & 0.06 & 0.01 \\
\hline Ca site tot & 2.97 & & 2.95 & & & 1.93 & 2.99 & 1.88 & & 1.91 & 2.95 & & \\
\hline $\mathbf{O}$ & 12.00 & 22.00 & 12.00 & 22.00 & 22.00 & 16.00 & 12.00 & 16.00 & 22.00 & 16.00 & 12.00 & 22.00 & 22.00 \\
\hline $\begin{array}{l}\mathrm{Fe} / \mathrm{Mg} \\
\% \mathrm{An}\end{array}$ & & 1.23 & 7.32 & 1.14 & & 16 & 8.95 & 27 & 1.93 & 30 & 10.20 & 1.69 & \\
\hline$-\ln K b-g$ & 1.78 & & 1.64 & & & & 1.53 & & & & 1.81 & & \\
\hline $\operatorname{avg} T, P$ & $570 \mathrm{C}, 2.7 \mathrm{~kb}$ & & & $590 \mathrm{C}, 4.2 \mathrm{~kb}$ & & & & $605 \mathrm{C}, 3.6 \mathrm{~kb}$ & & & 560C, $3.2 \mathrm{k}$ & & \\
\hline
\end{tabular}

See text for analytical methods. H2Oc=calculated water content; Ca site tot= \# cations in site containing $\mathrm{Ca}$; $\mathrm{O}=$ total oxygen per formula unit; T,P from techniques described in text; $\mathrm{b}=$ =biotite, $\mathrm{g}=$ garnet, $\mathrm{h}=$ hornblende, $\mathrm{p}=$ plagioclase, wm=white mica, -lnKb-g= negative log of (biotite)-Fe/Mg cations (garnet). 
Table 9 continued. Average microprobe data of mineral assemblages for geothermobarometric calculations.

\begin{tabular}{|c|c|c|c|c|c|c|c|c|c|c|c|}
\hline sample & ВК3-142-1 & BK3-142-1 & BK3-142-1 & BK3-142-2 & BK3- 142-2 & BK3-142-2 & 77-576-1 & 77-576-1 & $77-576-1$ & $77-576-2$ & $77-576-2$ \\
\hline mineral & $g$ & b & $\mathbf{p}$ & $g$ & b & $\mathbf{p}$ & $g$ & b & $\mathbf{p}$ & $g$ & b \\
\hline $\mathrm{Na2O}$ & 0.04 & 0.09 & 7.51 & 0.05 & 0.14 & 6.62 & 0.05 & 0.19 & 8.29 & 0.04 & 0.15 \\
\hline MgO & 2.42 & 7.18 & 0.01 & 2.21 & 6.92 & 0.01 & 2.14 & 7.99 & 0.01 & 2.38 & 7.96 \\
\hline Al203 & 22.07 & 19.85 & 25.93 & 21.72 & 19.85 & 27.63 & 21.73 & 21.25 & 24.93 & 21.70 & 21.78 \\
\hline SiO2 & 37.24 & 34.01 & 59.40 & 36.66 & 33.79 & 57.52 & 36.86 & 34.30 & 60.77 & 36.74 & 34.47 \\
\hline K2O & 0.01 & 9.40 & 0.38 & 0.03 & 9.65 & 0.20 & 0.05 & 9.49 & 0.33 & 0.04 & 9.78 \\
\hline $\mathrm{CaO}$ & 1.38 & 0.06 & 6.57 & 1.20 & 0.03 & 8.18 & 0.94 & 0.01 & 5.27 & 1.13 & 0.01 \\
\hline TiO2 & 0.03 & 2.43 & 0.02 & 0.05 & 2.64 & 0.02 & 0.00 & 1.88 & 0.05 & 0.06 & 1.29 \\
\hline $\mathrm{FeO}$ & 30.88 & 20.87 & 0.03 & 30.34 & 21.68 & 0.08 & 30.48 & 19.16 & 0.07 & 30.19 & 19.00 \\
\hline MnO & 7.42 & 0.33 & 0.03 & 8.19 & 0.41 & 0.05 & 8.86 & 0.19 & 0.04 & 7.92 & 0.23 \\
\hline H2Oc & & 3.85 & & & 3.86 & & & 3.90 & & & 3.91 \\
\hline Total & 101.51 & 98.08 & 99.88 & 100.45 & 98.99 & 100.30 & 101.10 & 98.37 & 99.76 & 100.21 & 98.59 \\
\hline \multicolumn{12}{|c|}{$\begin{array}{l}\begin{array}{l}\text { cations per } \\
\text { formula unit }\end{array} \\
\end{array}$} \\
\hline $\mathrm{Na}$ & 0.01 & 0.03 & 1.30 & 0.01 & 0.04 & 1.15 & 0.01 & 0.06 & 1.43 & 0.01 & 0.05 \\
\hline Mg & 0.29 & 1.66 & 0.00 & 0.27 & 1.60 & 0.00 & 0.26 & 1.83 & 0.00 & 0.29 & 1.82 \\
\hline Al & 2.07 & 3.64 & 2.73 & 2.06 & 3.63 & 2.90 & 2.06 & 3.84 & 2.62 & 2.06 & 3.93 \\
\hline Si & 2.96 & 5.29 & 5.30 & 2.96 & 5.24 & 5.13 & 2.96 & 5.26 & 5.41 & 2.96 & 5.28 \\
\hline $\mathbf{K}$ & 0.00 & 1.87 & 0.04 & 0.00 & 1.91 & 0.02 & 0.00 & 1.86 & 0.04 & 0.00 & 1.91 \\
\hline Ca & 0.12 & 0.01 & 0.63 & 0.10 & 0.01 & 0.78 & 0.08 & 0.00 & 0.50 & 0.10 & 0.00 \\
\hline $\mathbf{T i}$ & 0.00 & 0.28 & 0.00 & 0.00 & 0.31 & 0.00 & 0.00 & 0.22 & 0.00 & 0.00 & 0.15 \\
\hline $\mathbf{F e}$ & 2.06 & 2.72 & 0.00 & 2.05 & 2.81 & 0.01 & 2.05 & 2.46 & 0.01 & 2.04 & 2.43 \\
\hline Mn & 0.50 & 0.04 & 0.00 & 0.56 & 0.05 & 0.00 & 0.60 & 0.02 & 0.00 & 0.54 & 0.03 \\
\hline Ca site tot & 2.96 & & 1.93 & 2.98 & & 1.93 & 2.99 & & 1.93 & 2.97 & \\
\hline 0 & 12.00 & 22.00 & 16.00 & 12.00 & 22.00 & 16.00 & 12.00 & 22.00 & 16.00 & 12.00 & 22.00 \\
\hline $\mathrm{Fe} / \mathrm{Mg}$ & 7.15 & 1.63 & & 7.71 & 1.76 & & 8.01 & 1.35 & & 7.12 & 1.34 \\
\hline$\%$ An & & & 33 & & & 41 & & & 26 & & \\
\hline$-\ln K b-g$ & 1.48 & & & 1.48 & & & 1.78 & & & 1.67 & \\
\hline $\operatorname{avg} T, P$ & & 620C, $3.6 \mathrm{~kb}$ & & & $615 \mathrm{C}, 2.4 \mathrm{~kb}$ & & & 565C, $2.8 \mathrm{~kb}$ & & $580 \mathrm{C}, 3.2 \mathrm{~kb}$ & \\
\hline
\end{tabular}


Table 9 continued. Average microprobe data of mineral assemblages for geothermobarometric calculations.

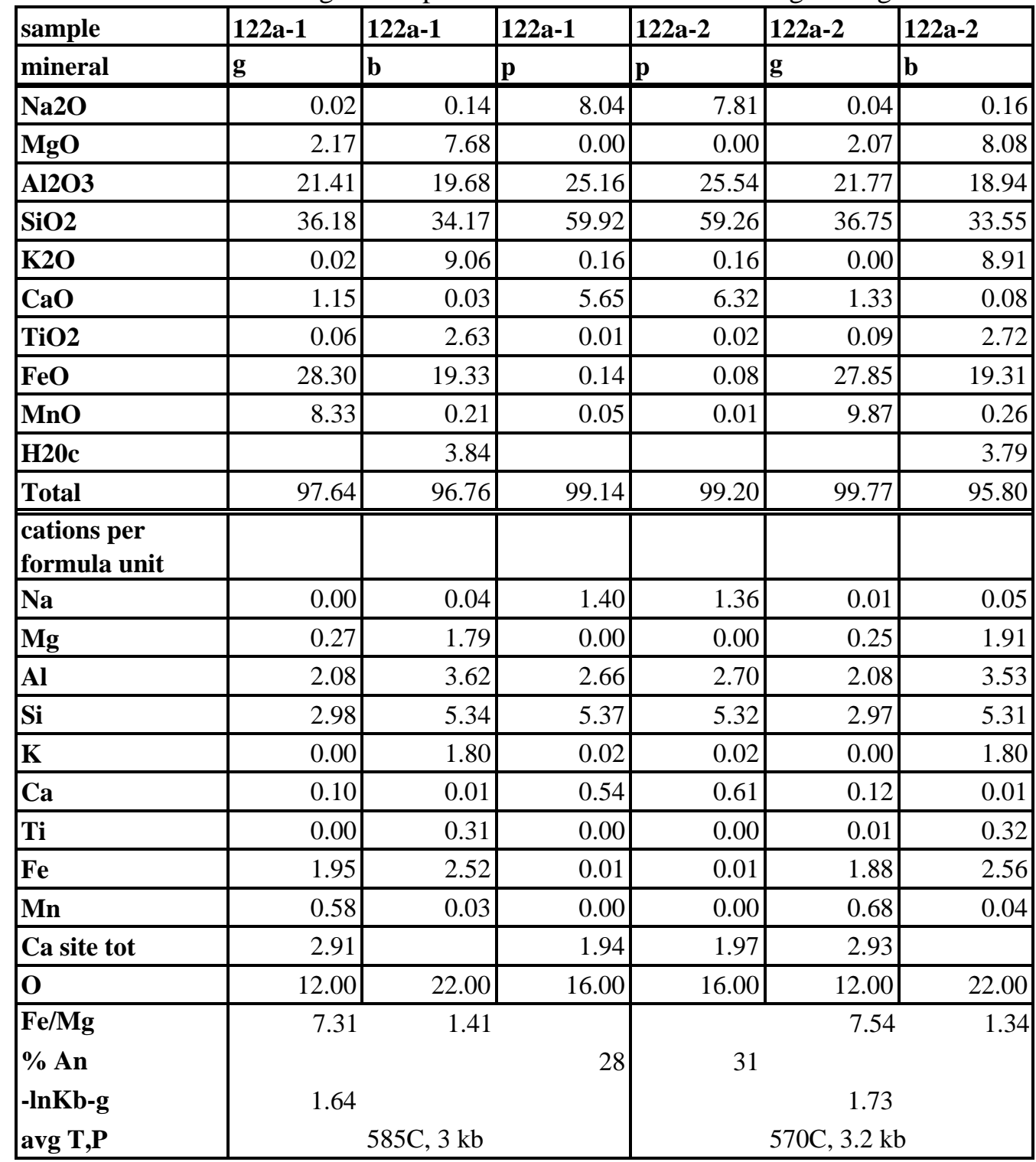


Table 10. Fluid inclusion samples, locations, and assay values of sampled interval.

\begin{tabular}{|c|c|c|c|c|c|}
\hline $\begin{array}{c}\text { Slide } \\
\text { (JGA-) }\end{array}$ & site & UTM E & UTM N & $\begin{array}{c}\text { Au Assay value } \\
\text { (ppm) }\end{array}$ & $\begin{array}{c}\text { Gold } \\
\text { Group }\end{array}$ \\
\hline $\mathbf{1 1}$ & BK2-047 (AuVn) & 7135800 & 536830 & $>1$ & 3 (high Au) \\
\hline $\mathbf{1 5}$ & BK2-152 (s15) & 7135800 & 536830 & 0.115 & 3 (high Au) \\
\hline $\mathbf{2}$ & BK1-65 & 7136400 & 536600 & .040 & 2 (interm. Au) \\
\hline $\mathbf{1}$ & BK1-035 & 7136400 & 536600 & 0.095 & 2 (interm. Au) \\
\hline $\mathbf{4}$ & BK1-082 & 7136400 & 536600 & $<0.02$ & 1 (Au not detected) \\
\hline $\mathbf{1 2}$ & BK2-012 & 7135800 & 536830 & $<0.02$ & 1 (Au not detected) \\
\hline $\mathbf{1 3}$ & BK2-135 & 7135800 & 536830 & $<0.02$ & 1 (Au not detected) \\
\hline B3 & $\begin{array}{c}\text { Buckeye Discovery } \\
\text { (Buckeye) }\end{array}$ & 7134900 & 536330 & up to 53 & 4 (Discovery high Au) \\
\hline
\end{tabular}


Table 11. Fluid inclusion data and estimated fluid parameters.

\begin{tabular}{|c|c|c|c|c|c|c|c|c|c|c|c|c|}
\hline Hole & Slide & Inclusion & $\mathrm{CO} 2$ & Clath & Cohom & Homog & \begin{tabular}{|l} 
Au group \\
\end{tabular} & Volume & Bars & Density & MoleH20 & Est. Press \\
\hline 1 & 1 & 2 & -60.2 & 12.4 & & 336 & 3 & 40 & & & & \\
\hline 1 & 1 & 3 & -62.3 & 13.3 & & 325 & 3 & 25 & & & & \\
\hline 1 & 1 & 4 & -61.5 & 12.6 & & 320 & 3 & 30 & & & & \\
\hline 1 & 1 & 5 & -58.3 & 12.5 & & 335 & 3 & 45 & & & & \\
\hline 1 & 1 & 6 & -61.5 & 13.6 & & 325 & 3 & 50 & & & & \\
\hline 1 & 1 & 7 & -57.3 & 10.9 & & 320 & 3 & 60 & & & & \\
\hline 1 & 1 & 8 & -62.9 & 14.2 & & 325 & 3 & 35 & & & & \\
\hline 1 & 2 & 1 & -58.7 & 10.6 & & 270 & 2 & 30 & & & & \\
\hline 1 & 2 & 2 & -57.5 & 11 & & 350 & 2 & 45 & & & & \\
\hline 1 & 2 & 3 & -58.6 & 12.7 & & 310 & 2 & 35 & & & & \\
\hline 1 & 2 & 4 & -57.5 & 11.5 & & 350 & 2 & 40 & & & & \\
\hline 1 & 2 & 5 & -57.8 & 11 & & 340 & 2 & 40 & & & & \\
\hline 1 & 2 & 6 & -57.5 & 10.6 & 23 & & 2 & 40 & 70 & 0.2 & 92 & 200 Bars \\
\hline 1 & 4 & 1 & -56.6 & 10.9 & & & 3 & 45 & & & & \\
\hline 1 & 4 & 2 & -56.6 & 10.4 & 27 & 340 & 3 & 45 & 70 & 0.23 & 90 & 200 Bars \\
\hline 1 & 4 & 3 & -56.7 & 10.8 & & 330 & 3 & 40 & & & & \\
\hline 1 & 4 & 4 & -57 & 10.6 & & 335 & 3 & 30 & & & & \\
\hline 1 & 4 & 5 & -56.9 & 10.9 & & 330 & 3 & 30 & & & & \\
\hline 2 & 11 & 1 & -57.3 & 10.9 & & 330 & 1 & 40 & & & & \\
\hline 2 & 11 & 2 & -57.5 & 10.8 & 25.9 & & 1 & 30 & 70 & 0.22 & 85 & 200 Bars \\
\hline 2 & 11 & 3 & -57.8 & 11.8 & 25.2 & & 1 & 25 & 70 & 0.22 & 95 & 200 Bars \\
\hline 2 & 11 & 4 & -57.6 & 10.9 & $25 *$ & & 1 & 45 & 150 & 0.78 & 82 & $>1 \mathrm{kbar}$ \\
\hline
\end{tabular}

* = CO2 homogenized to liquid $* *=$ critical value

Calculations are based on procedures discussed in Roedder (1984).

$\mathrm{CO} 2=$ Melting temperature of carbon dioxide in degree Celsius; Clath=Clathrate melting temperature in degree Celsius; Cohom=Partial homogenization of carbonic phase in degree Celsius; Homog=Total homogenization of carbonic phase in degree Celsius; Volume=Volume of vapor within inclusion shown in percent; Bars=Fluid inclusion pressure in bars after Angus and others, 1976; Density=CO2 density of the inclusion after Burress, 1981; $\mathrm{MoleH} 2 \mathrm{O}=$ Calculated mole percent of $\mathrm{H} 2 \mathrm{O}$ in inclusion fluids; Est. Press=Estimated minimum pressure at time of inclusion formation in bars. Employed P-T density isochores from Kennedy, 1954 and diagrams from Brown and Lamb, 1989. 
Table 11 continued. Fluid inclusion data and estimated fluid parameters.

\begin{tabular}{|c|c|c|c|c|c|c|c|c|c|c|c|c|}
\hline Hole & Slide & Inclusion & $\mathrm{CO} 2$ & Clath & Cohom & Homog & Au group & Volume & Bars & Density & Moleh20 & Est. Press \\
\hline 2 & 11 & 5 & -57.4 & 10.8 & $26.9 *$ & 280 & 1 & 50 & 150 & 0.76 & 80 & $>1 \mathrm{kbar}$ \\
\hline 2 & 11 & 6 & -61.4 & 11.1 & & & 1 & & & & & \\
\hline 2 & 11 & 7 & -58 & 11.5 & & 330 & 1 & 40 & & & & \\
\hline 2 & 11 & 9 & -58 & 11.7 & & 325 & 1 & 30 & & & & \\
\hline 2 & 11 & 10 & -59.1 & 10.6 & & 315 & 1 & 40 & & & & \\
\hline 2 & 11 & 11 & -58.1 & 11 & & & 1 & 30 & & & & \\
\hline 2 & 11 & 13 & -57 & 11.2 & & 310 & 1 & 20 & & & & \\
\hline 2 & 11 & 14 & -57 & 10.4 & & 310 & 1 & 25 & & & & \\
\hline 2 & 11 & 15 & -57.6 & 11.7 & & 310 & 1 & 35 & & & & \\
\hline 2 & 11 & 16 & -59.6 & 13.1 & & 295 & 1 & & & & & \\
\hline 2 & 12 & 1 & -58 & 11.8 & 19 & & 3 & 50 & 65 & 0.17 & 90 & 200 Bars \\
\hline 2 & 12 & 2 & -57.5 & 11.6 & 23 & 330 & 3 & 75 & 70 & 0.2 & 75 & 200 Bars \\
\hline 2 & 15 & 2 & -57 & 10.8 & 26.5 & $319 * *$ & 1 & 40 & 75 & 0.23 & 93 & 200 Bars \\
\hline 2 & 15 & 3 & -57 & 10.9 & 27 & 312.5 & 1 & 30 & 75 & 0.23 & 94 & 200 Bars \\
\hline 2 & 15 & 4 & -57 & 10.8 & & & 1 & 35 & & & & \\
\hline 2 & 15 & 5 & -56.6 & 10.7 & & 310 & 1 & & & & & \\
\hline 2 & 15 & 6 & -56.7 & 10.8 & 26.6 & & 1 & 40 & 75 & 0.23 & 95 & 200 Bars \\
\hline 2 & 15 & 9 & -57 & 10.8 & 30 & & 1 & 20 & 80 & 0.3 & 95 & 200 Bars \\
\hline 2 & 15 & 10 & -57 & 10.8 & & & 1 & 15 & & & & \\
\hline 2 & 15 & 11 & -56.6 & 10.3 & 29 & 300 & 1 & 30 & 75 & 0.25 & 92 & 200 Bars \\
\hline 2 & 15 & 12 & -56.6 & 10.5 & & 305 & 1 & 20 & & & & \\
\hline 2 & 15 & 13 & -56.8 & 10.9 & $28.5^{*}$ & & 1 & 35 & 120 & 0.75 & 82 & $>800$ bars \\
\hline \multicolumn{2}{|c|}{ Buckeye } & 1 & -65 & 15.6 & & 335 & & 60 & & & & \\
\hline
\end{tabular}


Table 11 continued. Fluid inclusion data and estimated fluid parameters.

\begin{tabular}{|c|c|c|c|c|c|c|c|c|c|c|c|c|}
\hline Hole & Slide & Inclusion & $\mathrm{CO} 2$ & Clath & Cohom & Homog & Au group & Volume & Bars & Density & Moleh20 & Est. Press \\
\hline \multicolumn{2}{|c|}{ Buckeye } & 3 & -57.6 & & & 330 & & 50 & & & & \\
\hline \multicolumn{2}{|c|}{ Buckeye } & 4 & -57.5 & 10.1 & & 330 & & 60 & & & & \\
\hline \multicolumn{2}{|c|}{ Buckeye } & 5 & -56.7 & 10.1 & & 340 & & 50 & & & & \\
\hline \multicolumn{2}{|c|}{ Buckeye } & 6 & 61.7 & 13.3 & & 335 & & 35 & & & & \\
\hline \multicolumn{2}{|c|}{ Buckeye } & 7 & -58.6 & 12.2 & 16.2 & 337 & & 35 & 50 & 0.17 & 94 & 200 Bars \\
\hline \multicolumn{2}{|c|}{ Buckeye } & 8 & -57.6 & 11.0 & 21.7 & 330 & & 35 & 60 & 0.19 & 95 & 200 Bars \\
\hline \multicolumn{2}{|c|}{ Buckeye } & 9 & -58.2 & 10.9 & 22.0 & 325 & & 50 & 60 & 0.19 & 90 & 200 Bars \\
\hline \multicolumn{2}{|c|}{ Buckeye } & 10 & -57.4 & 11.0 & 23.9 & 325 & & 35 & 68 & 0.2 & 94 & 200 Bars \\
\hline \multicolumn{2}{|c|}{ Buckeye } & 11 & -65.4 & 14.1 & & 350 & & 60 & & & & \\
\hline
\end{tabular}

Table 12. Comparison between Bald Knob, Democrat, Ryan Lode fluid inclusion data.

\begin{tabular}{|c|c|c|c|c|c|c|c|c|}
\hline Sample & $\begin{array}{c}\text { Size } \\
(\boldsymbol{\mu})\end{array}$ & $\begin{array}{c}\text { TmCO2 } \\
\left({ }^{\circ} \mathbf{C}\right)\end{array}$ & $\begin{array}{c}\text { Clathrate } \\
\mathbf{T} .\left({ }^{\circ} \mathbf{C}\right)\end{array}$ & $\begin{array}{c}\text { Th } \\
\left({ }^{\circ} \mathbf{C}\right)\end{array}$ & $\begin{array}{c}\text { Volume } \\
\text { \% vapor }\end{array}$ & $\begin{array}{c}\text { Salinity } \\
(\mathbf{\%} \text { NaCl) }\end{array}$ & $\begin{array}{c}\text { Mol \% } \\
\text { CO2 }\end{array}$ & $\begin{array}{c}\text { Trap P } \\
(\mathbf{b a r s})\end{array}$ \\
\hline Demo 1 & $20-60$ & -56.6 & not measured & $340-360$ & $10-90$ & $32-65$ & Variable & unknown \\
\hline Demo 2 & $50-100$ & -56.6 & not measured & $155-165$ & $10-90$ & $8-11.7$ & Variable & unknown \\
\hline BK hiP & $10-20$ & $-56.8-57.6$ & 10.9 & $\geq 280$ & $35-50$ & Low & 20 & $>1000$ \\
\hline BK loP & $10-20$ & $-56.6-62.9$ & $10.4-13.6$ & $300-345$ & $25-50$ & Low & 10 avg & $250-400$ \\
\hline Ryan Lode & $5-35$ & -56.6 to -58.4 & $9.3-12.5$ & $206-325$ & $10-90$ & Low & 12 & $250-300$ \\
\hline Bkeyel & $10-20$ & -61 to -65.4 & not measured & $330-350$ & $50-60$ & Low? & unknown & unknown \\
\hline Bkeye2 & $10-20$ & -57 to -65.4 & $10-15.5$ & $325-337$ & $35-50$ & Low & $5-10 \%$ & 250 \\
\hline
\end{tabular}

Demo 1, Demo 2 = Democrat (Pakhomova et al., 1995); BKhiP, BKloP = Bald Knob, high Pressure and low Pressure types (this study); Ryan Lode = Ryan Lode deposit, Fairbanks district (McCoy et al., 1997); Bkeye1,2 = Buckeye high grade vein (this study). 
Table 13. Interpreted ${ }^{40} \mathrm{Ar} /{ }^{39} \mathrm{Ar}$ ages (in Ma) for Richardson area samples.

\begin{tabular}{|l|l|l|l|l|}
\hline \multicolumn{1}{|c|}{$\begin{array}{c}\text { Sample number } \\
\text { (mineral) }\end{array}$} & $\begin{array}{c}\text { Low-temp fraction(s) } \\
\text { age (\% Ar) }\end{array}$ & $\begin{array}{c}\text { High-temp fraction(s) } \\
\text { age (\% Ar) }\end{array}$ & \multicolumn{1}{|c|}{ Plateau age (\% Ar) } & \multicolumn{1}{|c|}{ Interpreted age } \\
\hline $125(\mathrm{WM})$ & $87 \pm 32(1.5 \%)$ & $104.6 \pm 1.6(11.6 \%)$ & $102.0 \pm 0.5(81.4 \%)$ & $>105 \mathrm{Ma}$ \\
\hline $143(\mathrm{~B})$ & $80.5 \pm 1.6(19 \%)$ & $114.0 \pm 9(1.0 \%)$ & $92.2 \pm 0.6(76 \%)$ & $114 \pm 9 \mathrm{Ma}$ \\
\hline GRI-1 (B) & $56.9 \pm 3.3(0.6 \%)$ & $91.6 \pm 1.7(6.5 \%)$ & $90.7 \pm 0.9(78.3 \%)$ & $92 \pm 2 \mathrm{Ma}$ \\
\hline AuVn (WM) & excess Ar & $105.6+1.8(1.4 \%)$ & $104.8 \pm 1.0(51.4 \%)$ & $\sim 105 \mathrm{Ma}$ \\
\hline BR4-328 (WM) & $77.5+1.1(5.9 \%)$ & $96.6 \pm 8.7(0.8 \%)$ & $84.3 \pm 0.5(82.2 \%)$ & $85 \mathrm{Ma}<\mathrm{age}<97 \mathrm{Ma}$ \\
\hline
\end{tabular}

$(\mathrm{B})=$ biotite; $(\mathrm{WM})=$ White mica; $(\% \mathrm{Ar})=\%$ total ${ }^{39} \mathrm{Ar}$ released in the fraction(s). Spectrometer data in table 15.

Table 14. Ages of geologic events in the Richardson area as suggested by radiometric dating.

\begin{tabular}{|l|c|l|}
\hline \multicolumn{1}{|c|}{ Event } & Interpreted age & \multicolumn{1}{c|}{ Evidence } \\
\hline Cooling from peak metamorphism & $\sim 116 \mathrm{Ma}$ & $\begin{array}{l}\text { K-Ar age of hornblende from amphibolite } \\
\text { (Bundtzen and Reger, 1977) }\end{array}$ \\
\hline $\begin{array}{l}\text { Emplacement of peraluminous felsic } \\
\text { dikes following a collisional event }\end{array}$ & $\sim 114 \mathrm{Ma}$ & $\begin{array}{l}\text { Highest-temperature fraction age of sample } \\
\text { 143; regional correlations }\end{array}$ \\
\hline First Gold veining event & $\sim 105 \mathrm{Ma}$ & Sample AuVn; regional correlations \\
\hline Subduction-related magmatism & $\sim 92 \mathrm{Ma}$ & GRI-1 spectrum; regional correlations \\
\hline Second Gold veining event & $\sim 90 \mathrm{Ma}$ & Democrat lode deposit age; regional correlations \\
\hline Early Tertiary magmatism & $\sim 57 \mathrm{Ma}$ & $\begin{array}{l}\text { Post-90 Ma thermal resets to most spectra, } \\
\text { especially GRI-1 }\end{array}$ \\
\hline
\end{tabular}


Table $15 .{ }^{40} \mathrm{Ar} /{ }^{39} \mathrm{Ar}$ step-heating results and data.

\section{NOTES:}

Weighted average J (irradiation parameter) calculated from standard MMhb-1 (513.9 Ma)

Runs are step heat analyses of 1-3 single crystals of a mineral phase.

Laser power (in milliwatts) is the heating step from a defocussed argon-ion laser: $8700 \mathrm{~mW}$ represents the fusion step in most cases

$\%$ 39Ar: the proportion of 39Ar released in each step of a run.

Measured isotopic ratios (and 1-sigma error) are corrected for reactor induced interferences and decay of 37 $\mathrm{Ar}$ and 39Ar

$\%$ Atm. 40Ar*: percent of atmospheric 40Ar in the sample assuming an initial 40Ar/36Ar ratio of 295.5

$40 \mathrm{Ar} * / 39 \mathrm{ArK}(40 * / 39 \mathrm{~K})$ and ages (and 1-sigma error) calculated using the equations and constants quoted in McDougall and Harrison (1988)

\begin{tabular}{|c|c|c|c|c|c|c|c|c|c|c|c|c|c|}
\hline AF084- & R4-328 & I 02-1 & 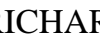 & & & ighted av & e of $\mathrm{J} f r$ & standa & .001380 & $+/-0$ & 0006 & & \\
\hline $\begin{array}{l}\text { Laser } \\
\text { Power }\end{array}$ & $\begin{array}{c}\text { Fraction } \\
39 \mathrm{Ar}\end{array}$ & $\begin{array}{c}\text { 40Ar/39Ar } \\
\text { measured }\end{array}$ & $+/-$ & $\begin{array}{c}37 \mathrm{Ar} / 39 \mathrm{Ar} \\
\text { measured }\end{array}$ & $+/-$ & $\begin{array}{c}36 \mathrm{Ar} / 39 \mathrm{Ar} \\
\text { measured }\end{array}$ & $+/-$ & $\begin{array}{l}\text { \% Atm. } \\
\text { 40Ar* }\end{array}$ & $\mathrm{Ca} / \mathrm{K}$ & $\mathrm{Cl} / \mathrm{K}$ & $40 * / 39 \mathrm{~K}$ & $\begin{array}{l}\text { Age } \\
\text { (Ma) }\end{array}$ & $\begin{array}{c}+/- \\
(\mathrm{Ma})\end{array}$ \\
\hline 0 & 0.059 & 40.506 & 0.366 & -0.0012 & 0.0033 & 0.0294 & 0.0013 & 21.4 & -0.002 & 0.0004 & 31.80 & 77.5 & 1.1 \\
\hline 150 & 0.098 & 33.700 & 0.229 & -0.0016 & 0.0057 & 0.0012 & 0.0015 & 1.1 & -0.003 & -0.0003 & 33.31 & 81.1 & 1.2 \\
\hline 200 & 0.159 & 33.581 & 0.227 & 0.0020 & 0.0059 & -0.0010 & 0.0021 & -0.9 & 0.004 & -0.0002 & 33.84 & 82.4 & 1.6 \\
\hline 300 & 0.260 & 33.915 & 0.272 & -0.0028 & 0.0016 & -0.0002 & 0.0005 & -0.2 & -0.005 & 0.0002 & 33.95 & 82.6 & 0.7 \\
\hline 600 & 0.518 & 35.631 & 0.204 & -0.0004 & 0.0011 & 0.0014 & 0.0005 & 1.1 & -0.001 & 0.0004 & 35.20 & 85.6 & 0.6 \\
\hline 750 & 0.691 & 35.324 & 0.195 & 0.0005 & 0.0015 & 0.0009 & 0.0005 & 0.8 & 0.001 & 0.0004 & 35.02 & 85.2 & 0.6 \\
\hline 900 & 0.748 & 34.413 & 0.266 & 0.0032 & 0.0054 & 0.0013 & 0.0017 & 1.1 & 0.006 & 0.0000 & 33.99 & 82.7 & 1.4 \\
\hline 1050 & 0.792 & 35.069 & 0.158 & -0.0005 & 0.0056 & 0.0014 & 0.0017 & 1.2 & -0.001 & 0.0001 & 34.63 & 84.2 & 1.2 \\
\hline 1200 & 0.837 & 35.506 & 0.175 & 0.0084 & 0.0046 & 0.0025 & 0.0018 & 2.1 & 0.015 & 0.0004 & 34.74 & 84.5 & 1.3 \\
\hline 1350 & 0.877 & 35.117 & 0.260 & -0.0033 & 0.0044 & 0.0023 & 0.0019 & 2.0 & -0.006 & 0.0004 & 34.40 & 83.7 & 1.5 \\
\hline 1500 & 0.920 & 34.083 & 0.254 & -0.0011 & 0.0049 & 0.0004 & 0.0016 & 0.3 & -0.002 & 0.0008 & 33.95 & 82.6 & 1.3 \\
\hline 2000 & 0.984 & 32.664 & 0.334 & 0.0066 & 0.0028 & 0.0015 & 0.0013 & 1.4 & 0.012 & 0.0007 & 32.18 & 78.4 & 1.2 \\
\hline 2500 & 0.992 & 37.260 & 0.794 & 0.0219 & 0.0180 & 0.0040 & 0.0100 & 3.2 & 0.040 & 0.0020 & 36.05 & 87.6 & 7.2 \\
\hline 3000 & 0.997 & 40.401 & 0.751 & -0.0388 & 0.0236 & -0.0005 & 0.0116 & -0.4 & -0.071 & 0.0018 & 40.52 & 98.2 & 8.3 \\
\hline 8000 & 1.000 & 42.068 & 2.344 & 0.0005 & 0.0743 & 0.0119 & 0.0279 & 8.4 & 0.001 & 0.0018 & 38.52 & 93.5 & 20.1 \\
\hline Integrated & & 35.170 & 0.080 & 0.0004 & 0.0009 & 0.0027 & 0.0003 & 2.3 & 0.001 & 0.0003 & 34.34 & 83.5 & 0.5 \\
\hline
\end{tabular}


Table 15 continued. ${ }^{40} \mathrm{Ar} /{ }^{39} \mathrm{Ar}$ step-heating results and data.

UAF084-54 AuVn WM 02-13-01 RICHARDSON Weighted average of J from standards $=0.001380+/-0.000006$

\begin{tabular}{|c|c|c|c|c|c|c|c|c|c|c|c|c|c|}
\hline $\begin{array}{l}\text { Laser } \\
\text { Power } \\
\end{array}$ & $\begin{array}{c}\text { Fraction } \\
\text { 39Ar }\end{array}$ & $\begin{array}{c}40 \mathrm{Ar} / 39 \mathrm{Ar} \\
\text { measured }\end{array}$ & $+/-$ & $\begin{array}{c}\text { 37Ar/39Ar } \\
\text { measured }\end{array}$ & $+/-$ & $\begin{array}{c}36 \mathrm{Ar} / 39 \mathrm{Ar} \\
\text { measured }\end{array}$ & $+/-$ & $\begin{array}{l}\% \text { Atm. } \\
\text { 40Ar* }\end{array}$ & $\mathrm{Ca} / \mathrm{K}$ & $\mathrm{Cl} / \mathrm{K}$ & $40 * / 39 \mathrm{~K}$ & $\begin{array}{l}\text { Age } \\
\text { (Ma) }\end{array}$ & $\begin{array}{r}+/- \\
(\mathrm{Ma}) \\
\end{array}$ \\
\hline 100 & 0.014 & 48.057 & 0.453 & 0.0091 & 0.0040 & 0.1409 & 0.0041 & 86.7 & 0.017 & 0.0014 & 6.41 & 15.9 & 2.9 \\
\hline 150 & 0.016 & 90.431 & 1.718 & -0.0289 & 0.0371 & 0.1641 & 0.0169 & 53.7 & -0.053 & 0.0004 & 41.89 & 101.4 & 11.8 \\
\hline 200 & 0.019 & 70.330 & 1.379 & -0.0042 & 0.0273 & 0.0743 & 0.0119 & 31.2 & -0.008 & -0.0026 & 48.35 & 116.6 & 8.5 \\
\hline 300 & 0.027 & 52.032 & 0.410 & 0.0075 & 0.0088 & 0.0262 & 0.0031 & 14.9 & 0.014 & 0.0010 & 44.26 & 107.0 & 2.3 \\
\hline 450 & 0.049 & 50.224 & 0.398 & -0.0023 & 0.0031 & 0.0195 & 0.0013 & 11.5 & -0.004 & 0.0006 & 44.44 & 107.4 & 1.3 \\
\hline 600 & 0.091 & 45.507 & 0.247 & -0.0013 & 0.0020 & 0.0088 & 0.0008 & 5.7 & -0.002 & 0.0005 & 42.89 & 103.8 & 0.8 \\
\hline 750 & 0.176 & 43.320 & 0.174 & -0.0022 & 0.0019 & 0.0022 & 0.0005 & 1.5 & -0.004 & 0.0006 & 42.63 & 103.1 & 0.5 \\
\hline 900 & 0.285 & 42.725 & 0.228 & 0.0000 & 0.0008 & 0.0009 & 0.0004 & 0.6 & 0.000 & 0.0006 & 42.42 & 102.7 & 0.6 \\
\hline 1050 & 0.400 & 42.917 & 0.238 & -0.0017 & 0.0007 & 0.0006 & 0.0003 & 0.4 & -0.003 & 0.0004 & 42.71 & 103.3 & 0.6 \\
\hline 1200 & 0.486 & 42.626 & 0.167 & 0.0002 & 0.0010 & 0.0012 & 0.0005 & 0.8 & 0.000 & 0.0006 & 42.25 & 102.3 & 0.5 \\
\hline 2000 & 0.758 & 43.874 & 0.487 & 0.0007 & 0.0004 & 0.0014 & 0.0001 & 1.0 & 0.001 & 0.0005 & 43.42 & 105.0 & 1.2 \\
\hline 8000 & 0.986 & 43.635 & 0.602 & -0.0001 & 0.0005 & 0.0012 & 0.0002 & 0.8 & 0.000 & 0.0005 & 43.25 & 104.6 & 1.4 \\
\hline 8700 & 1.000 & 45.057 & 0.389 & -0.0028 & 0.0038 & 0.0046 & 0.0022 & 3.0 & -0.005 & -0.0003 & 43.67 & 105.6 & 1.8 \\
\hline Integrated & & 43.949 & 0.196 & -0.0003 & 0.0003 & 0.0047 & 0.0001 & 3.1 & 0.000 & 0.0005 & 42.54 & 102.9 & 0.7 \\
\hline
\end{tabular}

UA F084-52 125 WM 02-13-01 RICHARDSON

Weighted average of $\mathrm{J}$ from standards $=0.001380+/-0.000006$

\begin{tabular}{|c|c|c|c|c|c|c|c|c|c|c|c|c|c|}
\hline $\begin{array}{l}\text { Laser } \\
\text { Power } \\
\end{array}$ & $\begin{array}{c}\text { Fraction } \\
\text { 39Ar }\end{array}$ & $\begin{array}{c}40 \mathrm{Ar} / 39 \mathrm{Ar} \\
\text { measured }\end{array}$ & $+/-$ & $\begin{array}{c}37 \mathrm{Ar} / 39 \mathrm{Ar} \\
\text { measured }\end{array}$ & $+/-$ & $\begin{array}{c}36 \mathrm{Ar} / 39 \mathrm{Ar} \\
\text { measured }\end{array}$ & $+/-$ & $\begin{array}{l}\text { \% Atm. } \\
\text { 40Ar* }\end{array}$ & $\mathrm{Ca} / \mathrm{K}$ & $\mathrm{Cl} / \mathrm{K}$ & $40 * / 39 \mathrm{~K}$ & $\begin{array}{l}\text { Age } \\
\text { (Ma) }\end{array}$ & $\begin{array}{c}+/- \\
(\mathrm{Ma}) \\
\end{array}$ \\
\hline 150 & 0.002 & 82.592 & 10.071 & -0.0025 & 0.2330 & 0.1381 & 0.1096 & 49.4 & -0.005 & -0.0060 & 41.77 & 101.1 & 76.3 \\
\hline 200 & 0.002 & 37.192 & 8.150 & -0.0883 & 0.1996 & 0.0743 & 0.1810 & 59.1 & -0.162 & -0.0281 & 15.19 & 37.4 & 130.2 \\
\hline 300 & 0.006 & 51.133 & 3.018 & 0.0824 & 0.0679 & 0.0648 & 0.0549 & 37.5 & 0.151 & 0.0062 & 31.96 & 77.9 & 38.9 \\
\hline 450 & 0.015 & 50.404 & 0.995 & 0.0155 & 0.0273 & 0.0319 & 0.0139 & 18.7 & 0.028 & -0.0011 & 40.95 & 99.2 & 9.9 \\
\hline 600 & 0.070 & 46.095 & 0.292 & -0.0145 & 0.0047 & 0.0084 & 0.0019 & 5.4 & -0.027 & 0.0014 & 43.57 & 105.4 & 1.5 \\
\hline 750 & 0.260 & 44.017 & 0.174 & -0.0062 & 0.0014 & 0.0051 & 0.0007 & 3.4 & -0.011 & 0.0004 & 42.48 & 102.8 & 0.6 \\
\hline 900 & 0.602 & 43.622 & 0.199 & -0.0028 & 0.0012 & 0.0051 & 0.0005 & 3.4 & -0.005 & 0.0004 & 42.10 & 101.9 & 0.6 \\
\hline 1050 & 0.884 & 42.898 & 0.169 & -0.0016 & 0.0009 & 0.0029 & 0.0003 & 2.0 & -0.003 & 0.0005 & 42.01 & 101.7 & 0.4 \\
\hline 1200 & 0.919 & 43.009 & 0.449 & -0.0153 & 0.0083 & -0.0034 & 0.0031 & -2.4 & -0.028 & 0.0005 & 43.99 & 106.4 & 2.4 \\
\hline 2000 & 0.977 & 43.055 & 0.333 & -0.0071 & 0.0078 & -0.0001 & 0.0024 & 0.0 & -0.013 & 0.0002 & 43.05 & 104.1 & 1.9 \\
\hline 8000 & 1.000 & 43.965 & 0.574 & 0.0090 & 0.0244 & 0.0044 & 0.0067 & 2.9 & 0.016 & 0.0002 & 42.65 & 103.2 & 4.8 \\
\hline Integrated & & 43.724 & 0.097 & -0.0038 & 0.0011 & 0.0047 & 0.0005 & 3.2 & -0.007 & 0.0005 & 42.30 & 102.4 & 0.6 \\
\hline
\end{tabular}


Table 15 continued. ${ }^{40} \mathrm{Ar} /{ }^{39} \mathrm{Ar}$ step-heating results and data.

UAF084-53 143 BI 02-14-01 RICHARDSON

Weighted average of $\mathrm{J}$ from standards $=0.001380+/-0.000006$

\begin{tabular}{|c|c|c|c|c|c|c|c|c|c|c|c|c|c|}
\hline $\begin{array}{l}\text { Laser } \\
\text { Power } \\
\end{array}$ & $\begin{array}{c}\text { Fraction } \\
\text { 39Ar }\end{array}$ & $\begin{array}{c}\text { 40Ar/39Ar } \\
\text { measured }\end{array}$ & $+/-$ & $\begin{array}{c}37 \mathrm{Ar} / 39 \mathrm{Ar} \\
\text { measured }\end{array}$ & $+/-$ & $\begin{array}{c}36 \mathrm{Ar} / 39 \mathrm{Ar} \\
\text { measured }\end{array}$ & $+/-$ & $\begin{array}{c}\text { \% Atm. } \\
\text { 40Ar* }\end{array}$ & $\mathrm{Ca} / \mathrm{K}$ & $\mathrm{Cl} / \mathrm{K}$ & $40 * / 39 \mathrm{~K}$ & $\begin{array}{l}\text { Age } \\
(\mathrm{Ma}) \\
\end{array}$ & $\begin{array}{c}+/- \\
(\mathrm{Ma}) \\
\end{array}$ \\
\hline 0 & 0.000 & -58.624 & 91.623 & 0.8905 & 3.5216 & 0.7584 & 1.8822 & -382.0 & 1.635 & 0.3167 & -282.86 & -892.4 & 2525.1 \\
\hline 150 & 0.190 & 36.220 & 0.650 & 0.0080 & 0.0024 & 0.0106 & 0.0007 & 8.6 & 0.015 & 0.0283 & 33.08 & 80.5 & 1.6 \\
\hline 200 & 0.370 & 38.493 & 0.224 & 0.0008 & 0.0031 & 0.0015 & 0.0008 & 1.1 & 0.001 & 0.0242 & 38.03 & 92.3 & 0.8 \\
\hline 300 & 0.519 & 38.055 & 0.215 & 0.0012 & 0.0028 & 0.0009 & 0.0008 & 0.7 & 0.002 & 0.0180 & 37.76 & 91.7 & 0.8 \\
\hline 450 & 0.718 & 38.434 & 0.377 & 0.0017 & 0.0028 & 0.0008 & 0.0009 & 0.6 & 0.003 & 0.0201 & 38.17 & 92.6 & 1.1 \\
\hline 600 & 0.869 & 38.003 & 0.308 & 0.0001 & 0.0030 & 0.0000 & 0.0011 & 0.0 & 0.000 & 0.0211 & 37.98 & 92.2 & 1.1 \\
\hline 750 & 0.926 & 38.229 & 0.271 & -0.0069 & 0.0088 & -0.0004 & 0.0026 & -0.3 & -0.013 & 0.0193 & 38.32 & 93.0 & 1.9 \\
\hline 900 & 0.950 & 37.771 & 0.385 & -0.0123 & 0.0194 & 0.0017 & 0.0060 & 1.4 & -0.022 & 0.0156 & 37.23 & 90.4 & 4.3 \\
\hline 1050 & 0.962 & 37.966 & 0.542 & -0.0394 & 0.0324 & -0.0144 & 0.0110 & -11.2 & -0.072 & 0.0133 & 42.20 & 102.1 & 7.8 \\
\hline 1200 & 0.972 & 37.980 & 0.731 & -0.0295 & 0.0433 & 0.0196 & 0.0166 & 15.3 & -0.054 & 0.0145 & 32.15 & 78.3 & 11.8 \\
\hline 1500 & 0.980 & 38.910 & 0.738 & -0.0096 & 0.0542 & 0.0071 & 0.0198 & 5.4 & -0.018 & 0.0152 & 36.78 & 89.3 & 13.9 \\
\hline 2000 & 0.991 & 38.251 & 0.559 & 0.0179 & 0.0244 & 0.0033 & 0.0096 & 2.5 & 0.033 & 0.0171 & 37.25 & 90.5 & 6.8 \\
\hline 8800 & 1.000 & 41.606 & 1.222 & -0.0027 & 0.0518 & -0.0192 & 0.0117 & -13.6 & -0.005 & 0.0108 & 47.24 & 114.0 & 8.7 \\
\hline Integrated & & 37.909 & 0.164 & 0.0007 & 0.0016 & 0.0024 & 0.0005 & 1.9 & 0.001 & 0.0218 & 37.17 & 90.3 & 0.7 \\
\hline
\end{tabular}

\begin{tabular}{|c|c|c|c|c|c|c|c|c|c|c|c|c|c|}
\hline $\begin{array}{l}\text { Laser } \\
\text { Power }\end{array}$ & $\begin{array}{c}\text { Fraction } \\
\text { 39Ar }\end{array}$ & $\begin{array}{c}\text { 40Ar/39Ar } \\
\text { measured }\end{array}$ & $+/-$ & $\begin{array}{c}\text { 37Ar/39Ar } \\
\text { measured }\end{array}$ & $+/-$ & $\begin{array}{c}36 \mathrm{Ar} / 39 \mathrm{Ar} \\
\text { measured }\end{array}$ & $+/-$ & $\begin{array}{l}\text { \% Atm. } \\
\text { 40Ar* }\end{array}$ & $\mathrm{Ca} / \mathrm{K}$ & $\mathrm{Cl} / \mathrm{K}$ & $40 * / 39 \mathrm{~K}$ & $\begin{array}{l}\text { Age } \\
(\mathrm{Ma})\end{array}$ & $\begin{array}{c}+/- \\
(\mathrm{Ma})\end{array}$ \\
\hline 150 & 0.006 & 37.907 & 0.636 & 0.1028 & 0.0144 & 0.0496 & 0.0044 & 38.7 & 0.189 & 0.0286 & 23.23 & 56.9 & 3.3 \\
\hline 200 & 0.019 & 39.946 & 0.255 & 0.0241 & 0.0078 & 0.0113 & 0.0028 & 8.3 & 0.044 & 0.0367 & 36.59 & 88.9 & 2.0 \\
\hline 300 & 0.076 & 37.656 & 0.171 & 0.0082 & 0.0010 & 0.0021 & 0.0005 & 1.6 & 0.015 & 0.0378 & 37.01 & 89.9 & 0.5 \\
\hline 450 & 0.221 & 37.430 & 0.439 & 0.0066 & 0.0006 & -0.0001 & 0.0002 & -0.1 & 0.012 & 0.0384 & 37.42 & 90.9 & 1.1 \\
\hline 600 & 0.364 & 37.589 & 0.504 & 0.0066 & 0.0011 & -0.0006 & 0.0005 & -0.5 & 0.012 & 0.0379 & 37.73 & 91.6 & 1.3 \\
\hline 750 & 0.544 & 37.470 & 0.855 & 0.0127 & 0.0009 & 0.0006 & 0.0003 & 0.5 & 0.023 & 0.0376 & 37.26 & 90.5 & 2.0 \\
\hline 900 & 0.802 & 37.497 & 0.730 & 0.0295 & 0.0006 & 0.0006 & 0.0003 & 0.4 & 0.054 & 0.0379 & 37.30 & 90.6 & 1.7 \\
\hline 1050 & 0.935 & 37.144 & 0.211 & 0.1014 & 0.0015 & -0.0005 & 0.0005 & -0.4 & 0.186 & 0.0383 & 37.27 & 90.5 & 0.6 \\
\hline 1200 & 0.980 & 37.649 & 0.221 & 0.3201 & 0.0044 & -0.0011 & 0.0015 & -0.9 & 0.587 & 0.0379 & 37.98 & 92.2 & 1.2 \\
\hline 1500 & 0.990 & 37.298 & 0.297 & 0.9425 & 0.0143 & -0.0004 & 0.0029 & -0.5 & 1.730 & 0.0342 & 37.48 & 91.0 & 2.1 \\
\hline 2000 & 0.996 & 37.261 & 0.359 & 0.5345 & 0.0238 & 0.0003 & 0.0053 & 0.1 & 0.981 & 0.0232 & 37.20 & 90.3 & 3.8 \\
\hline 8800 & 1.000 & 51.353 & 0.565 & 0.2672 & 0.0186 & 0.0502 & 0.0060 & 28.8 & 0.490 & 0.0142 & 36.53 & 88.7 & 4.3 \\
\hline Integrated & & 37.554 & 0.264 & 0.0549 & 0.0005 & 0.0008 & 0.0002 & 0.6 & 0.101 & 0.0377 & 37.29 & 90.5 & 0.8 \\
\hline
\end{tabular}




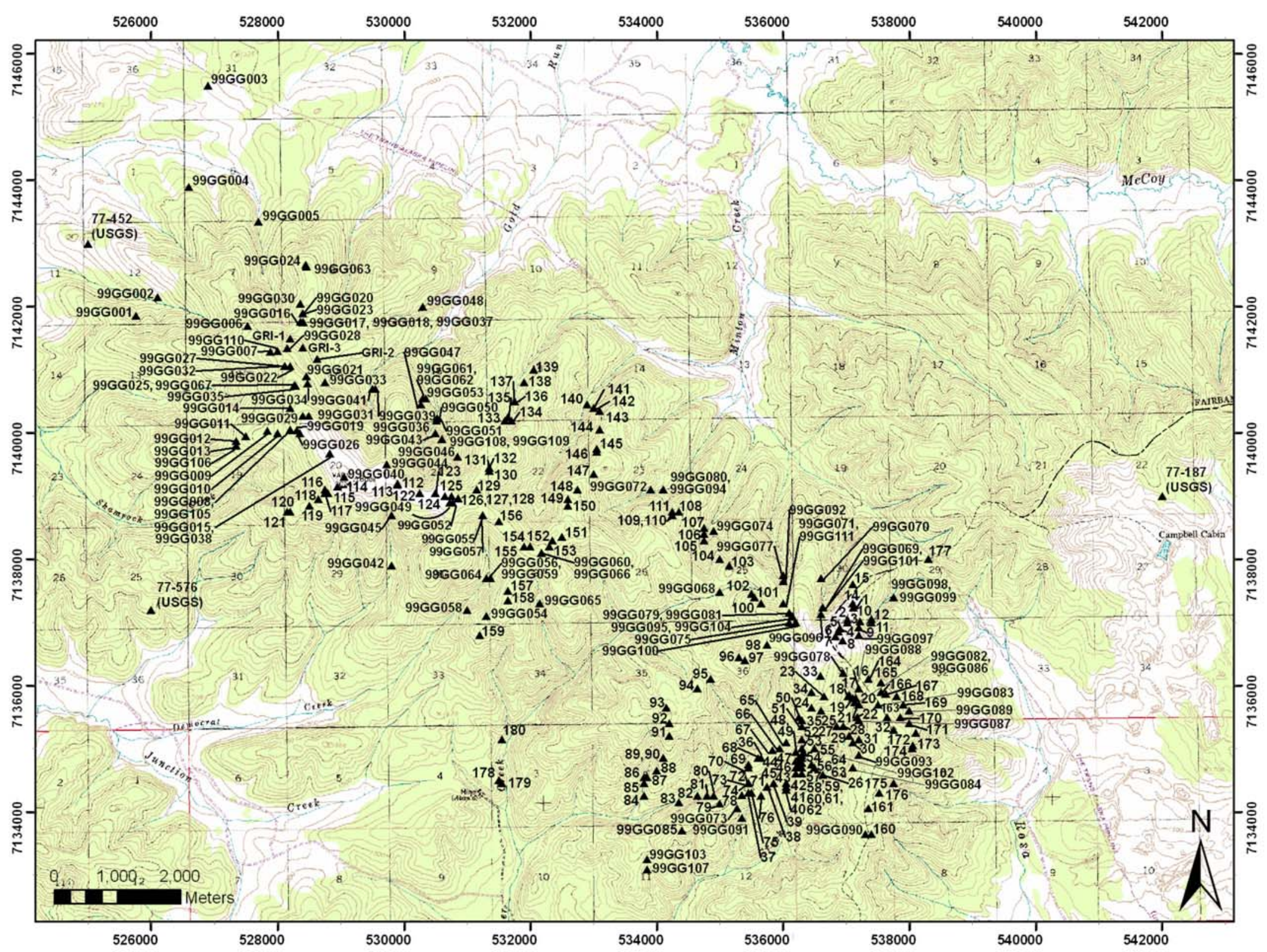

Figure 1. Location of analyzed samples from the Richardson district, Big Delta B-5 Quadrangle, Alaska. 


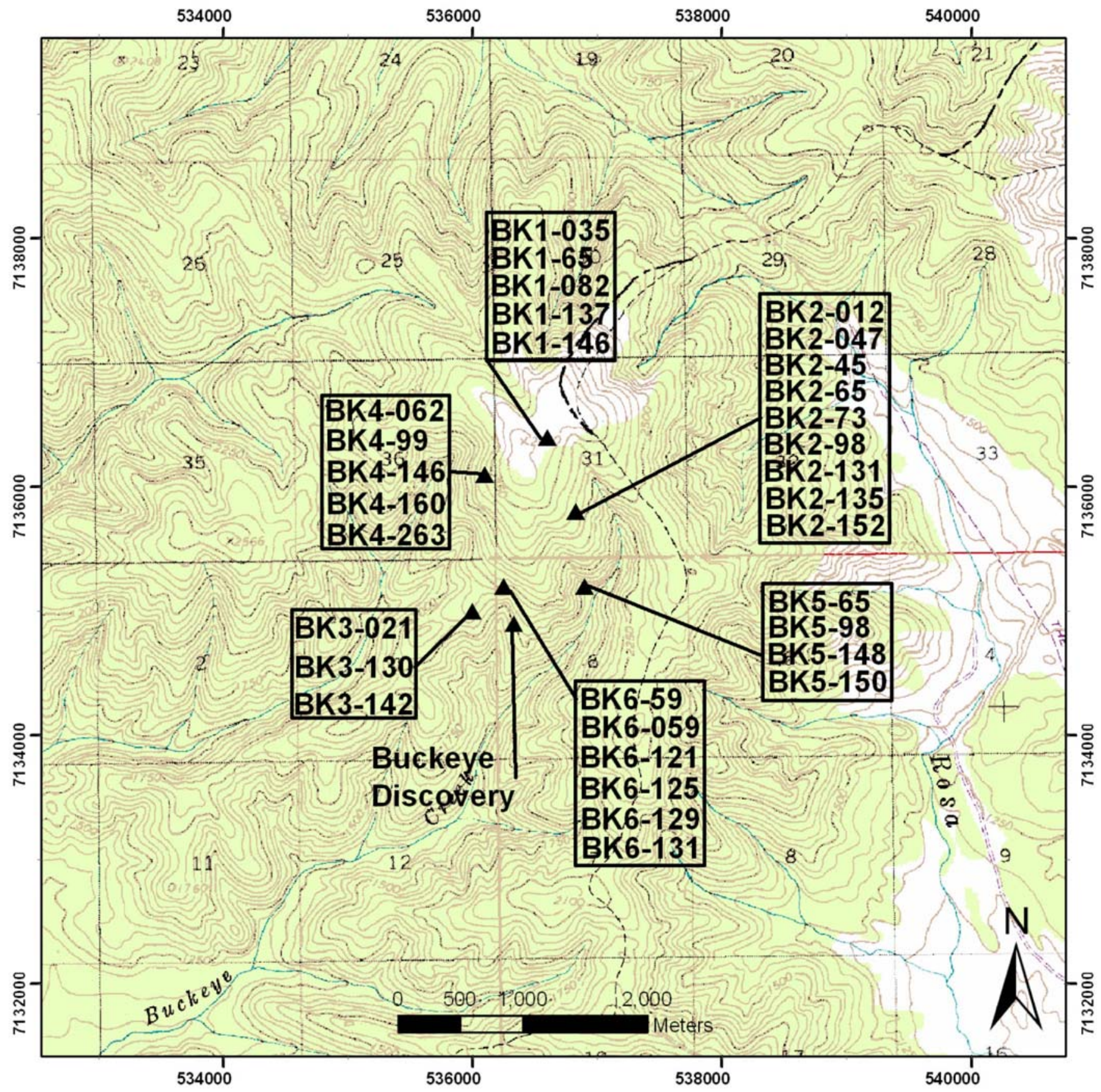

Figure 2. Location of Bald Knob drill hole samples from the Richardson district, Big Delta B-5 Quadrangle, Alaska. BK\# = drill hole number. 